Proc. Indian Acad. Sci. (Math. Sci.) Vol. 114, No. 2, May 2003, pp. 123-140.

Printed in India

\title{
Twisted holomorphic forms on generalized flag varieties
}

\author{
K PARAMASAMY \\ Chennai Mathematical Institute, Chennai 600 017, India \\ E-mail: paramas@cmi.ac.in \\ MS received 22 September 2003; revised 13 April 2004
}

\begin{abstract}
In this paper we prove some vanishing theorems for the twisted Dolbeault cohomology of the complete flag varieties associated to a simple, simply connected algebraic group.
\end{abstract}

Keywords. Simple algebraic group; flag variety; root system; cotangent sheaf.

\section{Introduction}

Let $G$ be a simple, simply connected algebraic group defined over an algebraically closed field $k$ of characteristic zero. Fix a maximal torus $T$. Let $W=\operatorname{Nor}_{G}(T) / \operatorname{Cent}_{G}(T)$ be the Weyl group and $X(T)=\operatorname{Hom}\left(T, G L_{1}(k)\right)$ be the group of characters. The main results of this article are as follows:

Theorem (Theorem 1). Let $G$ be a simple simply connected algebraic group over an algebraically closed field $k$ of characteristic zero. Let $p \geq 0$. Let $\lambda$ be a dominant weight such that for each $\mu \in \Phi_{p}^{-}$either $\lambda+\mu$ is dominant or $\lambda+\mu+\rho$ is singular. Then $H^{p, q}(G / B, \mathscr{L}(\lambda))=0$ for all $q \geq 1$.

Let $\lambda=\sum_{i} n_{i} \omega_{i}$. For given $p \geq 0$, in Proposition 2 we give conditions on $n_{i}$ 's so that $\lambda$ satisfies the hypothesis of Theorem 1.

For each simple root $\alpha$, we define the Coxeter number of $\alpha$, denoted by $h_{\alpha}$, as the number $\sum_{\gamma \in \Phi_{\alpha}^{+}}\left(\gamma, \alpha^{\vee}\right)$, where $\Phi_{\alpha}^{+}=\left\{\gamma \in \Phi^{+} \mid\left(\gamma, \alpha^{\vee}\right)>0\right\}$. We prove in Theorem 9 that $h_{\alpha} \leq h$, and for a shorter simple root $\beta, h_{\beta}=h$, where $h$ is the Coxeter number of $G$.

As a corollary of Theorem 1 and Proposition 2, we get the following vanishing theorem:

$$
H^{p, q}\left(G / B, \mathscr{L}_{\lambda}\right)=0, q \geq 1, p \geq 0, \text { if } n_{k} \geq h_{\alpha_{k}}-1, \forall k .
$$

Let $\operatorname{rank}(G) \geq 2$ and $\operatorname{dim}(G / B)=d$. In Theorem 12 we give strictly dominant weight $\lambda$ such that $H^{d-1,1}(G / B, \mathscr{L}(\lambda)) \neq 0$.

We also derive the following corollaries:

1. We show that the Bott vanishing property fails to hold for generalised flag varieties.

2. $G / B$ is not a toric variety.

3. $G / B$ cannot be degenerated to a smooth toric variety in such a manner that ample cone degenerates to ample cone. 
We recall some standard facts about algebraic groups. A standard reference for this material is [4]. For a finite dimensional $T$-module $V$ and $\chi \in X(T)$, set $V_{\chi}=\{v \in V \mid t v=$ $\chi(t) v, \forall t \in T\}$. The finitely many $\chi \in X(T)$ such that $V_{\chi} \neq 0$ are called weights of the $T$-module $V$ and $V_{\chi}$ the weight spaces. Then $V$ decomposes into a direct sum of weight spaces, i.e. $V=\bigoplus_{\chi_{i}} V_{\chi_{i}}$, where $\chi_{i}$ are weights.

Let $\mathfrak{g}$ and $\mathfrak{h}$ be the Lie algebras of $G$ and $T$ respectively. Let $T$ act on $\mathfrak{g}$ via the adjoint representation. The non-zero weights of this representation are called roots and we denote the set of all roots by $\Phi$. The weight space corresponding to the zero weight is $\mathfrak{h}$. We identify $X(T)$ canonically as a subset of $X(T) \otimes \mathbb{R}$. Let $\Delta$ denote the set of all simple roots, that is a subset of $\Phi$ such that (i) $\Delta$ is a basis of $X(T) \otimes \mathbb{R}$, (ii) for each root $\alpha \in \Phi$ there exist integers $n_{i}$ of like sign such that $\alpha=\sum n_{i} \alpha_{i}, \alpha_{i} \in \Delta$. The number of elements in $\Delta$ is called the rank of the group $G$. The set of all roots $\alpha \in \Phi$ for which the $n_{i}$ are not negative is denoted by $\Phi^{+}$and an element of $\Phi^{+}$is called a positive root. $\Phi^{-}:=\Phi-\Phi^{+}$is the set of negative roots. Let $\mathfrak{n}=\bigoplus_{\alpha \in \Phi^{+}} \mathfrak{g}_{\alpha}$ and $\mathfrak{n}^{-}=\bigoplus_{\alpha \in \Phi^{-}} \mathfrak{g}_{\alpha}$. Let $B$ be the Borel subgroup of $G$ such that the Lie algebra $\mathfrak{b}$ of $B$ is $\mathfrak{h} \oplus \mathfrak{n}^{-}$.

There is a natural faithful action of $W$ on $X(T) \otimes \mathbb{R}$ and there exists a $W$-invariant bilinear form $($,$) on X(T) \otimes \mathbb{R}$. For $\alpha \in \Delta$, we denote $2 \alpha /(\alpha, \alpha)$ by $\alpha^{\vee}$. For each $\alpha \in \Phi$, we have reflection $s_{\alpha} \in W$ defined by $s_{\alpha}(\psi)=\psi-\left(\psi, \alpha^{\vee}\right) \alpha, \forall \psi \in X(T) \otimes \mathbb{R}$. The set $\left\{s_{\alpha}, \alpha \in \Delta\right\}$ generates $W$. For each $\alpha_{i} \in \Delta$ there exists a weight $\omega_{i} \in X(T)$ such that $\left(\omega_{i}, \alpha_{j}^{\vee}\right)=\delta_{i j}$ (Here $\delta$ is the Krönecker delta.) These $\omega_{i}$ are called fundamental weights. The action of $W$ permutes the roots. For $w \in W$ we define the length of $w$, denoted by $l(w)$, to be the number of positive roots moved by $w$ to negative roots.

Following Jantzen [4], we denote the sheaf associated to a $B$-module $V$ by $\mathscr{L}(V)=$ $\mathscr{L}_{G / B}(V)$ (i.e. the sheaf of sections of the associated vector bundle on $\left.G / B\right)$. When $V=k_{\lambda}$, we denote $\mathscr{L}\left(k_{\lambda}\right)$ by $\mathscr{L}(\lambda)$. The tangent sheaf on $G / B([4]$, p. 229) is $\mathscr{L}(\mathfrak{g} / \mathfrak{b})$. The $T$ weights on $\mathfrak{g} / \mathfrak{b}$ are $\Phi^{+}$.

We say a weight $\lambda=\sum n_{k} \omega_{k}$ is dominant (strictly dominant) if $n_{k} \geq 0\left(n_{k}>0\right)$. We denote the set of dominant weight by $X(T)^{+}$. For $w \in W, \lambda \in X(T)$ we have the 'dot' action given by $w \cdot \lambda:=w(\lambda+\rho)-\rho$, where $\rho$ is half sum of positive roots. Now we recall the Borel-Weil-Bott theorem. Let $\lambda \in X(T)$. If $\lambda \in X(T)^{+}$then $H^{i}(G / B, \mathscr{L}(\lambda)) \neq 0$ only when $i=0$, and $\left\{H^{0}(G / B, \mathscr{L}(\lambda)) \lambda \in X(T)^{+}\right\}$is the set of all simple $G$-modules. If there is a $w \in W$ such that $w \cdot \lambda \in X(T)^{+}$(such a $w$ will be unique) then $H^{i}(G / B, \mathscr{L}(\lambda)) \neq 0$ only when $i=l(w)$ and $H^{l(w)}(G / B, \mathscr{L}(\lambda)) \simeq H^{0}(G / B, \mathscr{L}(w \cdot \lambda))$ and moreover if there is no such $w$ (equivalently if $\lambda+\rho$ is singular, i.e. $\left(\lambda+\rho, \alpha^{\vee}\right)=0$ for some root $\left.\alpha \in \Phi\right)$ then $H^{i}(G / B, \mathscr{L}(\lambda))=0, \forall i$.

Recall that in [3], Bott proved the following vanishing theorem for the complex projective space: $H^{*}\left(\mathbb{P}^{n}, \Omega_{\mathbb{P} n}^{q} \otimes \mathscr{O}(k)\right)=0,1 \leq k \leq q$. We say that a smooth projective variety $X$ satisfies the Bott vanishing property if the twisted holomorphic forms or Dolbeault cohomology $H^{p, q}(X, \mathscr{L})=H^{q}\left(X, \Omega^{p}(\mathscr{L})\right)=0$ for all ample line bundle $L, \forall q>0$ and $\forall p \geq 0$, where $\Omega^{p}$ stands for sheaf associated to the vector bundle $\Lambda^{p} T_{X}^{*}$ and $\Omega^{p}(\mathscr{L})=\Omega^{p} \otimes \mathscr{L}$.

We use the partial order ' $\geq$ ' in $X(T)$, for $\lambda_{1}, \lambda_{2} \in X(T)$, we say $\lambda_{1} \geq \lambda_{2}$ if $\lambda_{1}-\lambda_{2}=$ sum of some simple roots. For a simple algebraic group, the Coxeter number is defined as $\frac{\operatorname{dim}(G)}{\operatorname{rank}(G)}-1$. For the ordering of the simple roots we follow the following diagrams [5]. In the following diagram the arrows are pointing towards shorter simple root. If there is no arrow it means that the adjacent roots are of the same length. 


\section{Dynkin diagrams}

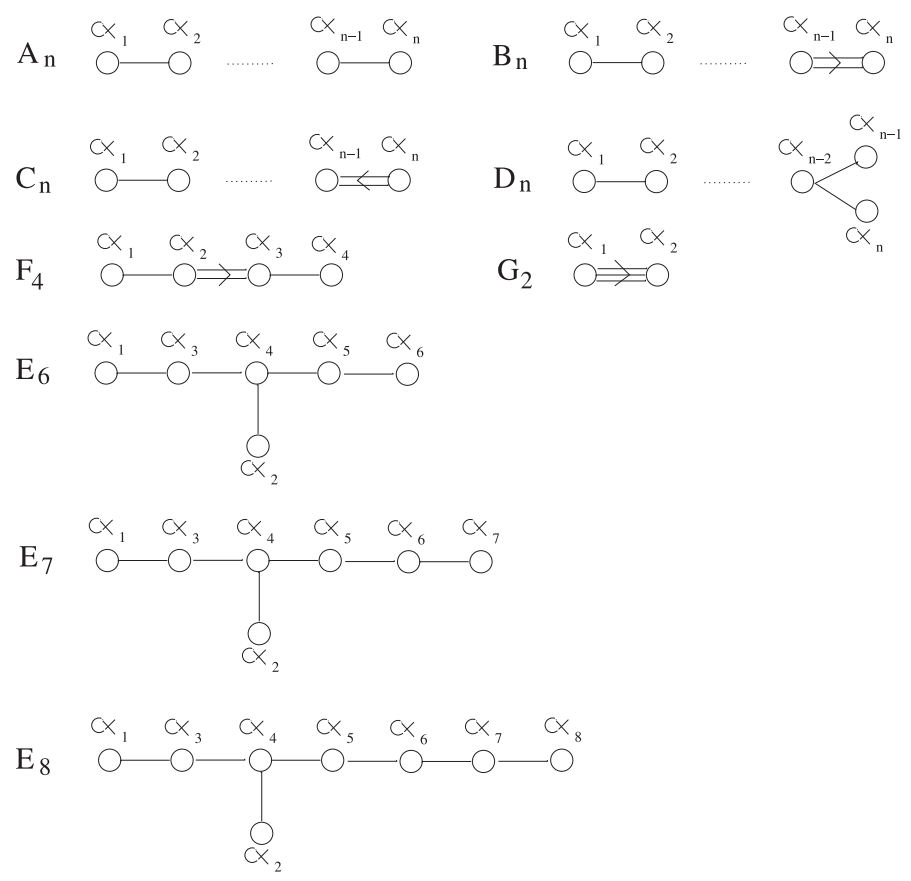

\subsection{New notations}

Now we introduce some new notations. For any subset $S$ of $X(T)$ and $j \geq 1, S_{j}:=\{v \in$ $X(T) \mid v=\sum_{i=1}^{j} \lambda_{i}, \lambda_{i} \in S$ and $\lambda_{i}$ 's are distinct $\}$. For any finite set $S$ of $X(T)$, we define an $|S| \times \operatorname{rank}(G)$-matrix associated to $S$ as follows: We represent $\lambda=\sum n_{k} \omega_{k} \in S$ as tuples $\left(n_{1}, n_{2}, \ldots\right)$, and write the elements of $S$ one below the other to get a $|S| \times \operatorname{rank}(G)$ matrix. We call the matrix corresponding to $\Phi^{+}$the positive roots matrix. For $\alpha \in \Delta, \Phi_{\alpha}^{+}:=\{\beta \in$ $\left.\Phi^{+} \mid\left(\beta, \alpha^{\vee}\right)>0\right\}$. We define the Coxeter number of $\alpha=h_{\alpha}=\sum_{\beta \in \Phi_{\alpha}^{+}}\left(\beta, \alpha^{\vee}\right)$.

\section{Vanishing theorems}

In this section, we study conditions on dominant weights $\lambda$ so that $H^{p, q}(G / B, \mathscr{L}(\lambda))=0$ for all $q \geq 1$ and $p \geq 0$.

Theorem 1. Let $p \geq 0$. Let $\lambda$ be a dominant weight such that for each $\mu \in \Phi_{p}^{-}$either $\lambda+\mu$ is dominant or $\lambda+\mu+\rho$ is singular. Then $H^{p, q}(G / B, \mathscr{L}(\lambda))=0$ for all $q \geq 1$.

Proof. The proof follows from the following observations:

1. By the hypothesis and by the Borel-Weil-Bott theorem, for any weight $v$ of $\Lambda^{p} \mathfrak{n}^{-} \otimes$ $k_{\lambda}$, we have $H^{q}(G / B, \mathscr{L}(v))=0$ for all $q \geq 1$.

2. Any lowest weight $\lambda_{1}$ of $\Lambda^{p} \mathfrak{n}^{-} \otimes k_{\lambda}$ will give a one-dimensional $B$-submodule $k_{\lambda_{1}}$. Let $V_{1}$ be $V / k_{\lambda_{1}}$. Then we will have a short exact sequence of $B$-modules; $0 \longrightarrow k_{\lambda_{1}} \longrightarrow$ $V \longrightarrow V_{1} \longrightarrow 0$. 
3. The above short exact sequence induces the following long exact sequence:

$$
\begin{aligned}
0 & \rightarrow H^{0}\left(G / B, \mathscr{L}\left(\lambda_{1}\right)\right) \rightarrow H^{p, 0}(G / B, \mathscr{L}(\lambda)) \rightarrow H^{0}\left(G / B, \mathscr{L}\left(V_{1}\right)\right) \\
& \rightarrow H^{1}\left(G / B, \mathscr{L}\left(\lambda_{1}\right)\right) \rightarrow H^{p, 1}(G / B, \mathscr{L}(\lambda)) \rightarrow H^{1}\left(G / B, \mathscr{L}\left(V_{1}\right)\right) \\
& \rightarrow H^{2}\left(G / B, \mathscr{L}\left(\lambda_{1}\right)\right) \rightarrow H^{p, 2}(G / B, \mathscr{L}(\lambda)) \rightarrow \cdots \cdots \cdots
\end{aligned}
$$

4. By 1 and 3, we have $H^{p, q}\left(G / B, \mathscr{L}_{\lambda}\right) \simeq H^{q}\left(G / B, \mathscr{L}\left(V_{1}\right)\right)$ for all $q \geq 1$. Now we repeat steps 2 and 3 with the $B$-module $V_{1}$. Let $\lambda_{2}$ be the lowest weight of $V_{1}$ and $V_{2}=V_{1} / k_{\lambda_{1}}$. As before we get $H^{q}\left(G / B, \mathscr{L}\left(V_{1}\right) \simeq H^{q}\left(G / B, \mathscr{L}\left(V_{2}\right)\right.\right.$ for all $q \geq 1$. After a finite number of steps we end up with a two-dimensional $B$-module $V_{j}$ such that $H^{q}\left(G / B, \mathscr{L}\left(V_{j}\right)\right)=0$ for all $q \geq 1$. This proves the theorem.

We now make explicit computations to give more delicate bound on the $\lambda$ for vanishing results. For this we use the classification of simple algebraic groups.

\section{PROPOSITION 2.}

(A) Let $G$ be a simply connected algebraic group of type $A_{n}$. Let $\lambda=\sum n_{k} \omega_{k}$. Then

$$
H^{p, q}(G / B, \mathscr{L}(\lambda))=0, \forall q \geq 1,
$$

if

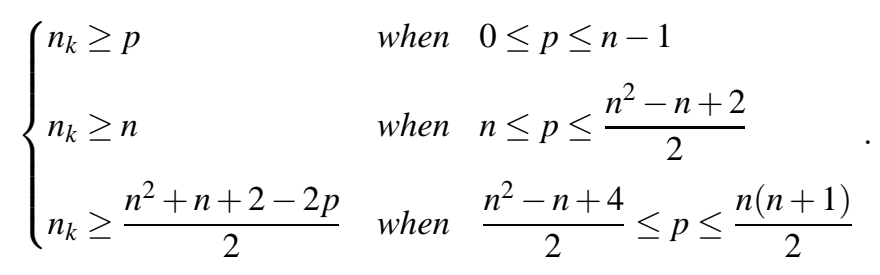

(B) Let $G$ be a simply connected algebraic group of type $B_{n}$. Let $\lambda=\sum n_{k} \omega_{k}$. Then

$$
H^{p, q}(G / B, \mathscr{L}(\lambda))=0, \forall q \geq 1,
$$

if

$$
\left\{\begin{array}{lll}
n_{k} \geq p, k \neq n ; n_{n} \geq 2 p-1 & \text { when } & 0 \leq p \leq n-1 \\
n_{k} \geq p, k \neq n ; n_{n} \geq 2 n-1 & \text { when } & n \leq p \leq 2 n-3 \\
n_{k} \geq 2 n-2, k \neq n ; n_{n} \geq 2 n-1 & \text { when } & 2 n-2 \leq p \leq n^{2}-2 n+3 \\
n_{k} \geq n^{2}+1-p, & \text { when } & n^{2}-2 n+4 \leq p \\
k \neq n ; n_{n} \geq 2 n-1 & & \leq n^{2}-n+1 \\
n_{k} \geq n^{2}+1-p, & \text { when } & n^{2}-n+2 \leq p \leq n^{2} \\
k \neq n ; n_{n} \geq 2 n^{2}+1-2 p & &
\end{array} .\right.
$$


(C) Let $G$ be a simply connected algebraic group of type $C_{n}$. Let $\lambda=\sum n_{k} \omega_{k}$. Then

$$
H^{p, q}(G / B, \mathscr{L}(\lambda))=0, \forall q \geq 1,
$$

if

$$
\left\{\begin{array}{lll}
n_{k} \geq 2 p-1, k \neq n ; n_{n} \geq p & \text { when } & p=1,2 \\
n_{k} \geq p+1, k \neq n ; n_{n} \geq p & \text { when } & 3 \leq p \leq n-1 \\
n_{k} \geq p+1, k \neq n ; n_{n} \geq n & \text { when } & n \leq p \leq 2 n-3 \\
n_{k} \geq 2 n-1, k \neq n ; n_{n} \geq n & \text { when } & 2 n-2 \leq p \leq n^{2}-2 n+3 \\
n_{k} \geq 2 n-3, k \neq n ; n_{n} \geq n & \text { when } & p=n^{2}-2 n+4 \\
n_{k} \geq n^{2}+1-p, & \text { when } & n^{2}-2 n+5 \\
k \neq n ; n_{n} \geq n & & \leq p \leq n^{2}-n+1 \\
n_{k} \geq n^{2}+1-p, & \text { when } & n^{2}-n+2 \leq p \leq n^{2} \\
k \neq n ; n_{n} \geq n^{2}+1-p & &
\end{array} .\right.
$$

(D) Let $G$ be a simply connected algebraic group of type $D_{n}$. Let $\lambda=\sum n_{k} \omega_{k}$. Then

$$
H^{p, q}(G / B, \mathscr{L}(\lambda))=0, \forall q \geq 1,
$$

if

$$
\left\{\begin{array}{lll}
n_{k} \geq p & \text { when } & 0 \leq p \leq 2 n-4 \\
n_{k} \geq 2 n-3 & \text { when } & 2 n-3 \leq p \leq n^{2}-3 n+4 \\
n_{k} \geq n^{2}-n+1-p & \text { when } & n^{2}-3 n+5 \leq p \leq n^{2}-n
\end{array}\right.
$$

$\left(\mathrm{E}_{6}\right)$ Let $G$ be a simply connected algebraic group of type $E_{6}$. Let $\lambda=\sum n_{k} \omega_{k}$. Then

$$
H^{p, q}(G / B, \mathscr{L}(\lambda))=0, \forall q \geq 1,
$$

if

$$
\left\{\begin{array}{lll}
n_{k} \geq p & \text { when } & 0 \leq p \leq 10 \\
n_{k} \geq 11 & \text { when } & 11 \leq p \leq 26 \\
n_{k} \geq 37-p & \text { when } & 27 \leq p \leq 36
\end{array}\right.
$$

(E) Let $G$ be a simply connected algebraic group of type $E_{7}$. Let $\lambda=\sum n_{k} \omega_{k}$. Then

$$
H^{p, q}(G / B, \mathscr{L}(\lambda))=0, \forall q \geq 1,
$$

if

$$
\left\{\begin{array}{lll}
n_{k} \geq p & \text { when } & 0 \leq p \leq 16 \\
n_{k} \geq 17 & \text { when } & 17 \leq p \leq 47 \\
n_{k} \geq 64-p & \text { when } & 48 \leq p \leq 63
\end{array} .\right.
$$


$\left(\mathrm{E}_{8}\right)$ Let $G$ be a simply connected algebraic group of type $E_{8}$. Let $\lambda=\sum n_{k} \omega_{k}$. Then

$$
H^{p, q}(G / B, \mathscr{L}(\lambda))=0, \forall q \geq 1,
$$

if

$$
\left\{\begin{array}{lll}
n_{k} \geq p & \text { when } & 0 \leq p \leq 28 \\
n_{k} \geq 29 & \text { when } & 29 \leq p \leq 92 \\
n_{k} \geq 121-p & \text { when } & 93 \leq p \leq 120
\end{array}\right.
$$

$\left(\mathrm{F}_{4}\right)$ Let $G$ be a simply connected algebraic group of type $F_{4}$. Let $\lambda=\sum n_{k} \omega_{k}$. Then

$$
H^{p, q}(G / B, \mathscr{L}(\lambda))=0, \forall q \geq 1,
$$

if

$$
\left\{\begin{array}{lll}
n_{1}, n_{2} \geq p ; n_{3}, n_{4} \geq 2 p-1 & \text { when } 0 \leq p \leq 4 \\
n_{1}, n_{2} \geq p ; n_{3}, n_{4} \geq p+3 & \text { when } & 5 \leq p \leq 7 \\
n_{1}, n_{2} \geq 8 ; n_{3}, n_{2} \geq 11=h_{k}-1 & \text { when } 8 \leq p \leq 17 \\
n_{1}, n_{2} \geq 25-p ; & \text { when } 18 \leq p \leq 20 \\
n_{3}, n_{4} \geq 45-2 p & & \\
n_{k} \geq 25-p & \text { when } & 21 \leq p \leq 24
\end{array} .\right.
$$

$\left(\mathrm{G}_{2}\right)$ Let $G$ be a simply connected algebraic group of type $G_{2}$. Let $\lambda=n \omega_{1}+m \omega_{2}$. Then

$$
H^{p, q}(G / B, \mathscr{L}(\lambda))=0, \forall q \geq 1
$$

if

$$
\begin{cases}n, m \geq 1 & \text { when } p=1,6 \\ n \geq 2, m \geq 4 & \text { when } p=2,5 . \\ n \geq 3, m \geq 5 & \text { when } p=3,4\end{cases}
$$

Proof. We consider the set of weights of $\Lambda^{p} \mathfrak{n}^{-} \otimes k_{\lambda}$. By our notation this set is $\Phi_{p}^{-}+\{\lambda\}$. Our aim is to make all $v \in \Phi_{p}^{-}+\{\lambda\}$ either dominant or $\lambda+v$ singular for suitable choice of $\lambda$. For that we give condition on $\left(\lambda, \alpha_{i}^{\vee}\right)$ so that $\left(\nu, \alpha_{i}^{\vee}\right) \geq-1$ for all $\alpha_{i} \in \Delta$. To avoid complications we include only simple singularities, i.e. we give conditions on $\left(\lambda, \alpha_{i}^{\vee}\right)$ so that either $v \in X(T)^{+}$, or there exists a simple root $\alpha$ such that $\left(v+\rho, \alpha^{\vee}\right)=0$.

If we know in each column of the 'positive roots matrix', what the positive entries, the negative entries, and the zeros are, and how many times they appear, then we can compute easily what the maximum positive value is which can occur in $\left(\mu, \alpha_{i}^{\vee}\right)$ for all $\alpha_{i}^{\vee} \in \Delta$, where $\mu$ runs over $\Phi_{p}^{+}$. Suppose that this number is $n_{i}$, then we demand that $\lambda$ satisfy the inequalities $\left(\lambda, \alpha_{i}^{\vee}\right) \geq n_{i}-1 \forall i$. 
The following observation will be useful in writing down the conditions stated in the proposition. In the positive root matrix, suppose there are $m_{1}$ positive entries and $m_{2}$ negative entries and $m_{3}$ zeros in the $i$ th column. Then the maximum among the positive entries in the $i$ th column of the matrix corresponding to $\Phi_{p}^{+}$increases when $p$ increases from zero to $m_{1}$ (how quickly it increases depends on what the positive entries are (with repetition)); it will be non-decreasing when $p$ increases from $m_{1}$ to $m_{1}+m_{3}$, and then it starts decreasing (again, how quickly it decreases depends on how many negative entries there are (with repetition)).

We give the needed information about the positive roots matrix in the following table.

\begin{tabular}{cc|ccccccc}
\hline & & 0 & 1 & 2 & 3 & -1 & -2 & -3 \\
\hline$A_{n}$ & & $\frac{n^{2}-3 n+2}{2}$ & $n-1$ & 1 & 0 & $n-1$ & 0 & 0 \\
$B_{n}$ & Short & $n^{2}-2 n+1$ & 0 & $n$ & 0 & 0 & $n-1$ & 0 \\
& Long & $n^{2}-4 n+5$ & $2 n-3$ & 1 & 0 & $2 n-3$ & 0 & 0 \\
$C_{n}$ & Short & $n^{2}-4 n+5$ & $2 n-4$ & 2 & 0 & $2 n-4$ & 1 & 0 \\
& Long & $n^{2}-2 n+1$ & $n-1$ & 1 & 0 & $n-1$ & 0 & 0 \\
$D_{n}$ & & $n^{2}-5 n+7$ & $2 n-4$ & 1 & 0 & $2 n-4$ & 0 & 0 \\
$E_{6}$ & & 15 & 10 & 1 & 0 & 10 & 0 & 0 \\
$E_{7}$ & & 30 & 16 & 1 & 0 & 16 & 0 & 0 \\
$E_{8}$ & & 63 & 28 & 1 & 0 & 28 & 0 & 0 \\
$F_{4}$ & Short & 9 & 4 & 4 & 0 & 4 & 3 & 0 \\
& Long & 9 & 7 & 1 & 0 & 7 & 0 & 0 \\
$G_{2}$ & Short & 1 & 1 & 1 & 1 & 1 & 0 & 1 \\
& Long & 1 & 2 & 1 & 0 & 2 & 0 & 0 \\
\hline
\end{tabular}

Remark 3. We do not present all the computations of the table mainly for want of space. However, we give some computations for classical groups in the next section. We give explicitly the matrix corresponding to $\Phi^{+}$for all the exceptional groups in Appendix A.

Remark 4. Let $\lambda=\sum n_{k} \omega_{k}$. We gave conditions on $n_{k}$ 's depending on $p$ so as to have $H^{p, q}(G / B, \mathscr{L}(\lambda))=0, \forall q \geq 1$. But conversely the vanishing $H^{p, q}(G / B, \mathscr{L}(\lambda))=0, \forall q \geq$ 1 need not imply that the $n_{k}$ 's satisfy such conditions. For example let $G$ be of type $E_{6}$ and let $p=\operatorname{dim}(G / B)-1$. If $\lambda=2 \rho-\omega_{2}$ or $\lambda=\rho$ then $\lambda$ will not satisfy the conditions given in the theorem but $H^{p, q}(G / B, \mathscr{L}(\lambda))=0, \forall q \geq 1$.

\section{COROLLARY 5.}

$H^{p, q}(G / B, \mathscr{L}(\lambda))=0, \forall q \geq 1, \forall p \geq 0$, when $n_{k} \geq h_{\alpha_{k}}-1, \forall k$.

Proof. It follows from Proposition 2 and Theorem 1.

The following corollary is a weaker version of the above corollary. 


\section{COROLLARY 6.}

$H^{p, q}(G / B, \mathscr{L}(\lambda))=0, \forall q \geq 1, \forall p \geq 0$, when $n_{k} \geq h-1, \forall k$.

Remark 7. We could directly prove Corollary 6 along the same lines of the proof of Theorem 1 with the following observations. This is due to Andersen and Jantzen [1]. Let $v$ be a weight of $\Lambda^{j} \mathfrak{n}^{-}$, for some $j$. Then $\max _{\alpha \in \Phi}\left|\left(v+\rho, \alpha^{\vee}\right)\right| \leq h-1$.

Proof. Recall that $\rho$ is the half sum of positive roots. Since $v$ is the sum of some distinct negative roots, we have $v+\rho=\frac{1}{2} \sum_{\alpha \in S} \alpha-\frac{1}{2} \sum_{\alpha \in S^{\prime}} \alpha$ where $S^{\prime} \subset \Phi^{+}$such that $v=$ $\sum_{\alpha \in S^{\prime}}-\alpha$, and $S=\Phi^{+}-S^{\prime}$. Since $W$ acts on $\Phi, w(v+\rho)$ will be of the above form and hence $w(v+\rho) \leq \rho, \forall w \in W$. Now fix $w$ such that $w(v+\rho) \in X(T)^{+}$.

One has

$$
\begin{aligned}
\max _{\alpha \in \Phi}\left|\left(v+\rho, \alpha^{\vee}\right)\right| & =\max _{\alpha \in \Phi}\left|\left(w(v+\rho), \alpha^{\vee}\right)\right| \\
& =\max _{\alpha \in \Phi}\left(w(v+\rho), \alpha^{\vee}\right) \leq \max _{\alpha \in \Phi}\left(\rho, \alpha^{\vee}\right)=h-1
\end{aligned}
$$

which gives the inequality quoted in the above remark.

\section{A remark on the Coxeter number}

From the above table, we can easily obtain the following lemma.

\section{Lemma 8.}

(a) If $\alpha_{i}, \alpha_{j} \in \Delta$ such that $\left|\alpha_{i}\right|=\left|\alpha_{j}\right|$ then $h_{\alpha_{i}}=h_{\alpha_{j}}$.

(b) If $\alpha_{i}, \alpha_{j} \in \Delta$ such that $\left|\alpha_{i}\right| \leq\left|\alpha_{j}\right|$ then $h_{\alpha_{i}} \geq h_{\alpha_{j}}$.

Proof. As we mentioned, the proof can be read off from the table. However, we give a conceptual proof for (a). Let $\alpha \in \Phi^{+}$. For any $\gamma \in \Phi^{+}-\{\alpha\}$, consider the $\alpha$-string $\gamma-$ $p \alpha, \ldots, \gamma+q \alpha$ through $\gamma$, where $p-q=\left(\gamma, \alpha^{\vee}\right)$. It follows that $\left(\gamma-(p-i) \alpha, \alpha^{\vee}\right)=-p-$ $q+2 i=-\left(\gamma+(q-i) \alpha, \alpha^{\vee}\right)$. Since $\gamma \in \Phi^{+}-\{\alpha\}$, is in a unique $\alpha$-string of maximum length, we have $h_{\alpha}=\sum_{\gamma \in \Phi_{\alpha}^{+}}\left(\gamma, \alpha^{\vee}\right)=\frac{1}{2}\left(2+\sum_{\gamma \in \Phi^{+}}\left|\left(\gamma, \alpha^{\vee}\right)\right|\right)=\frac{1}{4}\left(4+\sum_{\gamma \in \Phi}\left|\left(\gamma, \alpha^{\vee}\right)\right|\right)$. Now since the pairing $($,$) is W$-invariant and since $W$ acts transitively on the set of roots of $G$ of the same length, (a) follows.

Theorem 9. Let $\bar{h}$ be $\max _{\alpha \in \Delta}\left\{h_{\alpha}\right\}$. The number $\bar{h}$ coincides with the Coxeter number $h$.

Proof. The proof we give uses the Dynkin classification. The proof follows from the table. Since we have not given computation of the table, here we give the proof for certain cases in classical type. Appendix A gives complete proofs for all the exceptional groups.

1. $A_{n}$ type

Any root $\gamma \in \Phi^{+}$is a linear combination of simple roots with coefficient 0 or 1 . Let $\left\{\alpha_{1}, \alpha_{2}, \ldots, \alpha_{n}\right\}$ be a set of simple roots (with the usual order). We write the positive roots as an ordered linear combination of simple roots (i.e. if $\alpha_{1}$ appears in the linear combination then it should appear first and then $\alpha_{2}$ and so on). Let $\alpha_{i}$ be a simple root. It is clear that for $\gamma \in \Phi^{+},\left(\gamma, \alpha_{i}^{\vee}\right)>0$ (i.e. when we write $\gamma$ in terms of fundamental weights, the coefficient of $\omega_{i}$ is strictly positive) iff $\gamma$ begins with $\alpha_{i}$ or ends with $\alpha_{i}$. 
The number of positive roots beginning with $\alpha_{i}$ is $n+1-i$ (the roots are $\alpha_{i}, \alpha_{i}+$ $\left.\alpha_{i+1}, \ldots, \alpha_{i}+\alpha_{i+1}+\cdots+\alpha_{n}\right)$, and the number of positive roots ending with $\alpha_{i}$ is $i$ (the roots are $\left.\alpha_{i}, \alpha_{i-1}+\alpha_{i}, \ldots, \alpha_{1}+\alpha_{2}+\cdots+\alpha_{i}\right)$. Note that we have counted $\alpha_{i}$ twice. Therefore the total number of positive roots with $\omega_{i}$ th coefficient strictly positive is $n$. Among all such positive roots, only $\alpha_{i}$ will have $\omega_{i}$ th coefficient to be 2 , all other positive roots will have coefficient to be 1 . So $h_{\alpha_{i}}=n+1, \forall i$, hence $\bar{h}=n+1=h$.

2. $B_{n}$ type

We compute $h_{\alpha_{n}}$. The positive roots with $\omega_{n}$ th coefficient strictly positive are $\alpha_{n}, \alpha_{n-1}+2 \alpha_{n}, \alpha_{n-2}+\alpha_{n-1}+2 \alpha_{n}, \ldots, \alpha_{1}+\cdots+\alpha_{n-1}+2 \alpha_{n}$, and they all will have $\omega_{n}$ th coefficient 2 , hence $\bar{h}=h_{\alpha_{n}}=2 n=h$.

3. $C_{n}$ type

We compute $h_{\alpha_{1}}$. The positive roots with $\omega_{1}$ th coefficient strictly positive are $\alpha_{1}, \alpha_{1}+\alpha_{2}, \alpha_{1}+\alpha_{2}+\alpha_{3}, \ldots, \alpha_{1}+\cdots+\alpha_{n}$ (total number is $n$ ), and $\alpha_{1}+\alpha_{2}+2 \alpha_{3}+$ $\cdots+2 \alpha_{n-1}+\alpha_{n}, \alpha_{1}+\alpha_{2}+\alpha_{3}+2 \alpha_{4}+\cdots+2 \alpha_{n-1}+\alpha_{n}, \ldots, \alpha_{1}+\alpha_{2}+\cdots+\alpha_{n-2}+$ $2 \alpha_{n-1}+\alpha_{n}$ (total number is $n-3$, and $2 \alpha_{1}+2 \alpha_{2}+\cdots+2 \alpha_{n-1}+\alpha_{n}$. All of them will have $\omega_{1}$ th coefficient 1 , except $\alpha_{1}$ and $2 \alpha_{1}+2 \alpha_{2}+\cdots+2 \alpha_{n-1}+\alpha_{n}$, and $\omega_{1}$ th coefficient of these two weights will be 2 , hence $\bar{h}=h_{\alpha_{n}}=2 n=h$.

4. $D_{n}$ type

We compute $h_{\alpha_{1}}$. The positive roots with $\omega_{1}$ th coefficient strictly positive are $\alpha_{1}, \alpha_{1}+$ $\alpha_{2}, \alpha_{1}+\alpha_{2}+\cdots+\alpha_{n}$ (total number is $n$ ), and $\alpha_{1}+\alpha_{2}+\cdots+\alpha_{n-2}+\alpha_{n}$, and $\alpha_{1}+$ $\alpha_{2}+2 \alpha_{3}+\cdots+2 \alpha_{n-2}+\alpha_{n-1}+\alpha_{n}, \alpha_{1}+\alpha_{2}+\alpha_{3}+2 \alpha_{4}+\cdots+2 \alpha_{n-2}+\alpha_{n-1}+$ $\alpha_{n}, \ldots, \alpha_{1}+\alpha_{2}+\cdots+\alpha_{n-3}+2 \alpha_{n-2}+\alpha_{n-1}+\alpha_{n}$ (total number is $\left.n-4\right)$. All of them will have $\omega_{1}$ th coefficient 1 except $\alpha_{1}$, and $\alpha_{1}$ will have 2 , and hence $h_{\alpha_{1}}=\bar{h}=$ $2 n-2=h$.

\section{Remarks on Bott vanishing property}

In this section we prove that the Bott vanishing property fails to hold on generalised flag varieties. More precisely, we prove that for a simple, simply connected algebraic group $G$ of rank $\geq 2$, there exists ample line bundle $L_{\lambda}$ with $H^{d-1,1}(G / B, \mathscr{L}(\lambda)) \neq 0$, where $d=\operatorname{dim}(G / B)$. Since $G / B$ is defined over $\mathbb{Z}$, the non-vanishing results hold even in prime characteristic for large primes.

Lemma 10. Let $V$ be a finite dimensional B-module with a one-dimensional Bsubmodule $k_{\lambda}$ and let $U$ be the quotient module $V / k_{\lambda}$. If $\lambda \in X(T)-W \cdot X(T)^{+}$then $H^{i}(G / B, \mathscr{L}(V)) \simeq H^{i}(G / B, \mathscr{L}(U)), \forall i$.

Proof. Consider the short exact sequence of $B$-modules $0 \rightarrow K_{\lambda} \rightarrow V \rightarrow U \rightarrow 0$. This induces a long exact sequence of cohomology modules

$$
\begin{aligned}
0 & \rightarrow H^{0}(G / B, \mathscr{L}(\lambda)) \rightarrow H^{0}(G / B, \mathscr{L}(V)) \rightarrow H^{0}(G / B, \mathscr{L}(U)) \\
& \rightarrow H^{1}(G / B, \mathscr{L}(\lambda)) \rightarrow H^{1}(G / B, \mathscr{L}(V)) \rightarrow H^{1}(G / B, \mathscr{L}(U)) \\
& \rightarrow H^{2}(G / B, \mathscr{L}(\lambda)) \rightarrow H^{2}(G / B, \mathscr{L}(V)) \rightarrow H^{2}(G / B, \mathscr{L}(U)) \rightarrow \cdots
\end{aligned}
$$

By the Borel-Weil-Bott theorem we know that $H^{i}(G / B, \mathscr{L}(\lambda))=0$ for all $i$. 
Lemma 11.

(i) Let $G$ be simple group with $\operatorname{rank}(G) \geq 4$, other than type $F_{4}$ and $D_{n}$. Let $\lambda=2 \rho-\alpha_{n-2}-\alpha_{n-1}$. Then $\lambda$ is strictly dominant and the weights of $\Lambda^{d-1} \mathfrak{n}^{-} \otimes k_{\lambda}$ all lie in $X(T)-W \cdot X(T)^{+}$except $-\alpha_{n-1},-\alpha_{n-2}, 0$. More precisely, if $\mu$ is $a$ weight of $\Lambda^{d-1} \mathfrak{n}^{-} \otimes k_{\lambda}$ and $\mu \notin\left\{-\alpha_{n-2},-\alpha_{n-1}, 0\right\}$, then there exists $a$ $v \in\left\{\alpha_{n-2}, \alpha_{n-1}, \alpha_{n-2}+\alpha_{n-1}\right\}$ such that $\left(\mu+\rho, v^{\vee}\right)=0$.

(ii) Let $G$ be a simple group of rank 2 or rank 3 or of type $F_{4}$. Let $\lambda=2 \rho-\alpha_{1}-\alpha_{2}$. Then $\lambda$ is strictly dominant and the weights of $\Lambda^{d-1} \mathfrak{n}^{-} \otimes k_{\lambda}$ all lie in $X(T)-W$. $X(T)^{+}$except $-\alpha_{2},-\alpha_{1}, 0$ (for $G_{2}$, in addition to this we will have $(0,1)$ also as a non-singular weight). More precisely, if $\mu$ is a weight of $\Lambda^{d-1} \mathfrak{n}^{-} \otimes k_{\lambda}$ and $\mu \notin$ $\left\{-\alpha_{1},-\alpha_{2}, 0\right\}$, then there exists a $v \in\left\{\alpha_{1}, \alpha_{2}, \alpha_{1}+\alpha_{2}\right\}$ such that $\left(\mu+\rho, v^{\vee}\right)=0$.

(iii) Let $G$ be $D_{n}$ type. Let $\lambda=2 \rho-\alpha_{n-3}-\alpha_{n-2}$. Then $\lambda$ is strictly dominant and the weights of $\Lambda^{d-1} \mathfrak{n}^{-} \otimes k_{\lambda}$ all lie in $X(T)-W \cdot X(T)^{+}$except $-\alpha_{n-2},-\alpha_{n-3}, 0$. More precisely, if $\mu$ is a weight of $\Lambda^{d-1} \mathfrak{n}^{-} \otimes k_{\lambda}$ and $\mu \notin\left\{-\alpha_{n-3},-\alpha_{n-2}, 0\right\}$, then there exists a $v \in\left\{\alpha_{n-3}, \alpha_{n-2}, \alpha_{n-3}+\alpha_{n-2}\right\}$ such that $\left(\mu+\rho, v^{\vee}\right)=0$.

Proof. For the exceptional groups, we could verify the lemma directly from the tables given in the Appendix.

For the classical groups we essentially need to prove the statements for rank 4 and rank 5 groups for $D_{n}$ type, for other types rank $\leq 4$ groups. For these groups it is very easy to check it by hand. For the convenience of the reader we give the table of positive roots in these cases. Then we can easily check the statements.

\begin{tabular}{|c|c|c|c|c|c|c|c|c|c|c|c|c|c|c|c|c|}
\hline & $A_{4}$ & & & & & & $B_{4}$ & & & & & & $C_{4}$ & & & \\
\hline$\left(\begin{array}{llll}1 & 0 & 0 & 0\end{array}\right) \leftrightarrow$ & $(2$ & -1 & 0 & 0) & $\left(\begin{array}{llll}1 & 0 & 0 & 0\end{array}\right)$ & $\leftrightarrow$ & $(2$ & -1 & 0 & 0) & $\left(\begin{array}{llll}1 & 0 & 0 & 0\end{array}\right)$ & ans & ( 2 & -1 & 0 & 0) \\
\hline$\left(\begin{array}{llll}0 & 1 & 0 & 0\end{array}\right)$ « & $(-1$ & 2 & -1 & 0) & $\left(\begin{array}{llll}0 & 1 & 0 & 0\end{array}\right)$ & ans & $(-1$ & 2 & -1 & 0) & $\left(\begin{array}{llll}0 & 1 & 0 & 0\end{array}\right)$ & ans & $(-1$ & 2 & -1 & 0) \\
\hline$\left(\begin{array}{llll}0 & 0 & 1 & 0\end{array}\right)$ & $(0$ & -1 & 2 & $-1)$ & $\left(\begin{array}{llll}0 & 0 & 1 & 0\end{array}\right)$ & $\leftrightarrow$ & $(0$ & -1 & 2 & $-2)$ & $\left(\begin{array}{llll}0 & 0 & 1 & 0\end{array}\right)$ & trat & $(0$ & -1 & 2 & $-1)$ \\
\hline$\left(\begin{array}{llll}0 & 0 & 0 & 1\end{array}\right) \leftrightarrow$ & $(0$ & 0 & -1 & 2) & $\left(\begin{array}{llll}0 & 0 & 0 & 1\end{array}\right)$ & tus & $(0$ & 0 & -1 & 2) & $\left(\begin{array}{llll}0 & 0 & 0 & 1\end{array}\right)$ & ans & $(0$ & 0 & -2 & 2) \\
\hline (1) 1000$)$ & $(1$ & 1 & -1 & $0)$ & $\left(\begin{array}{llll}1 & 1 & 0 & 0\end{array}\right)$ & tha & $(1$ & 1 & -1 & $0)$ & $\left(\begin{array}{llll}1 & 1 & 0 & 0\end{array}\right)$ & ans & ( 1 & 1 & -1 & $0)$ \\
\hline (0) 1010$)$ & $(-1$ & 1 & 1 & $-1)$ & $\left(\begin{array}{llll}0 & 1 & 1 & 0\end{array}\right)$ & thl & $(-1$ & 1 & 1 & $-2)$ & $\left(\begin{array}{llll}0 & 1 & 1 & 0\end{array}\right)$ & trus & $(-1$ & 1 & 1 & $-1)$ \\
\hline$\left(\begin{array}{lllll}0 & 0 & 1 & 1\end{array}\right) \leftrightarrow$ & $(0$ & -1 & 1 & 1) & $\left(\begin{array}{llll}0 & 0 & 1 & 1\end{array}\right)$ & $\leftrightarrow x$ & $(0$ & -1 & 1 & $0)$ & $\left(\begin{array}{llll}0 & 0 & 1 & 1\end{array}\right)$ & ans & $(0$ & -1 & 0 & 1) \\
\hline (1) 1110$)$ & $(1$ & 0 & 1 & $-1)$ & $\left(\begin{array}{llll}1 & 1 & 1 & 0\end{array}\right)$ & $\leftrightarrow$ & $(1$ & 0 & 1 & $-2)$ & $\left(\begin{array}{llll}1 & 1 & 1 & 0\end{array}\right)$ & ans & $(1$ & 0 & 1 & $-1)$ \\
\hline$\left(\begin{array}{llll}0 & 1 & 1 & 1\end{array}\right) \longleftrightarrow \leadsto$ & $(-1$ & 1 & 0 & 1) & $\left(\begin{array}{llll}0 & 1 & 1 & 1\end{array}\right)$ & xns & $(-1$ & 1 & 0 & 0) & $\left(\begin{array}{llll}0 & 1 & 1 & 1\end{array}\right)$ & ans & $(-1$ & 1 & -1 & 1) \\
\hline (1) & $(1$ & 0 & 0 & 1) & $\left(\begin{array}{llll}0 & 0 & 1 & 2\end{array}\right)$ & that & $(0$ & -1 & 0 & 2) & $\left(\begin{array}{llll}0 & 0 & 2 & 1\end{array}\right)$ & $\leftrightarrow$ & $(0$ & -2 & 2 & 0) \\
\hline & & & & & $\left(\begin{array}{llll}1 & 1 & 1 & 1\end{array}\right)$ & tha & $(1$ & 0 & 0 & 0) & $\left(\begin{array}{llll}1 & 1 & 1 & 1\end{array}\right)$ & ans & ( 1 & 0 & -1 & 1) \\
\hline & & & & & $\left(\begin{array}{llll}0 & 1 & 1 & 2\end{array}\right)$ & tha & $(-1$ & 1 & -1 & 2) & $\left(\begin{array}{llll}0 & 1 & 2 & 1\end{array}\right)$ & 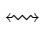 & $(-1$ & 0 & 1 & 0) \\
\hline & & & & & $\left(\begin{array}{llll}1 & 1 & 1 & 2\end{array}\right)$ & tus & $(1$ & 0 & -1 & 2) & $\left(\begin{array}{llll}1 & 1 & 2 & 1\end{array}\right)$ & ans & ( 1 & -1 & 1 & 0) \\
\hline & & & & & $\left(\begin{array}{llll}0 & 1 & 2 & 2\end{array}\right)$ & tha & $(-1$ & 0 & 1 & $0)$ & $\left(\begin{array}{llll}0 & 2 & 2 & 1\end{array}\right)$ & ans & $(-2$ & 2 & 0 & $0)$ \\
\hline & & & & & $\left(\begin{array}{llll}1 & 1 & 2 & 2\end{array}\right)$ & thl & $(1$ & -1 & 1 & 0) & $\left(\begin{array}{llll}1 & 2 & 2 & 1\end{array}\right)$ & trus & $(0$ & 1 & 0 & $0)$ \\
\hline & & & & & $\left(\begin{array}{llll}1 & 2 & 2 & 2\end{array}\right)$ & $\leftrightarrow$ & $(0$ & 1 & 0 & 0) & $\left(\begin{array}{llll}2 & 2 & 2 & 1\end{array}\right)$ & that & $(2$ & 0 & 0 & 0) \\
\hline
\end{tabular}

\begin{tabular}{|c|c|c|c|c|c|c|c|c|c|c|c|c|c|c|c|}
\hline \multicolumn{3}{|c|}{$B_{2}$} & \multicolumn{4}{|c|}{$A_{3}$} & \multicolumn{4}{|c|}{$B_{3}$} & \multicolumn{5}{|c|}{$C_{3}$} \\
\hline (1) & ( 2 & $-2)$ & (1) & $(2$ & -1 & 0) & (1) & $(2$ & -1 & 0) & $\left(\begin{array}{lll}1 & 0 & 0\end{array}\right)$ & ans & ( 2 & -1 & 0) \\
\hline (0 $\left.\begin{array}{ll}0 & 1\end{array}\right)$ & $(-1$ & 2) & $\left(\begin{array}{lll}0 & 1 & 0\end{array}\right)$ & $(-1$ & 2 & $-1)$ & $\left(\begin{array}{lll}0 & 1 & 0\end{array}\right)$ & $(-1$ & 2 & $-2)$ & $\left(\begin{array}{lll}0 & 1 & 0\end{array}\right)$ & ans & $(-1$ & 2 & $-1)$ \\
\hline (1 1 ) & ( 1 & $0)$ & $\left(\begin{array}{lll}0 & 0 & 1\end{array}\right)$ thr & $(0$ & -1 & 2) & $\left(\begin{array}{lll}0 & 0 & 1\end{array}\right)$ & $(0$ & -1 & 2) & $\left(\begin{array}{lll}0 & 0 & 1\end{array}\right)$ & tans & ( 0 & -2 & 2) \\
\hline (1 2) (2) & $(0$ & 2) & the & $(1$ & 1 & $-1)$ & (1) & ( 1 & 1 & $-2)$ & $\left(\begin{array}{lll}1 & 1 & 0\end{array}\right)$ & turs & ( 1 & 1 & $-1)$ \\
\hline & & & $\left.\begin{array}{lll}0 & 1 & 1\end{array}\right)$ & $(-1$ & 1 & 1) & $\left.\begin{array}{lll}0 & 1 & 1\end{array}\right)$ & $(-1$ & 1 & $0)$ & $\left(\begin{array}{lll}0 & 1 & 1\end{array}\right)$ & ans & $(-1$ & 0 & 1) \\
\hline & & & (1) 1111$)$ & ( 1 & 0 & 1) & (1) & ( 1 & 0 & 0) & $\left(\begin{array}{lll}1 & 1 & 1\end{array}\right)$ & tans & ( 1 & -1 & 1) \\
\hline & & & & & & & $\left(\begin{array}{lll}0 & 1 & 2\end{array}\right)$ & $(-1$ & 0 & 2) & $\left(\begin{array}{lll}0 & 2 & 1\end{array}\right)$ & tans & $(-2$ & 2 & 0) \\
\hline & & & & & & & (1) & ( 1 & -1 & 2) & $\left(\begin{array}{lll}1 & 2 & 1\end{array}\right)$ & thrs & ( 0 & 1 & $0)$ \\
\hline & & & & & & & (1) & $(0$ & 1 & 0) & $\left(\begin{array}{lll}2 & 2 & 1\end{array}\right)$ & thrs & ( 2 & 0 & $0)$ \\
\hline
\end{tabular}




\begin{tabular}{|c|c|c|c|c|c|c|c|c|c|c|c|c|}
\hline & & $D_{4}$ & & & & & & & $D_{5}$ & & & \\
\hline$\left(\begin{array}{llll}1 & 0 & 0 & 0\end{array}\right)$ & $\leftrightarrow$ & $(2$ & -1 & 0 & 0) & $\left(\begin{array}{lllll}1 & 0 & 0 & 0 & 0\end{array}\right)$ & ans & $(2$ & -1 & 0 & 0 & 0) \\
\hline$\left(\begin{array}{llll}0 & 1 & 0 & 0\end{array}\right)$ & $\leftrightarrow$ & $(-1$ & 2 & -1 & $-1)$ & $\left(\begin{array}{lllll}0 & 1 & 0 & 0 & 0\end{array}\right)$ & that & $(-1$ & 2 & -1 & 0 & 0) \\
\hline$\left(\begin{array}{llll}0 & 0 & 1 & 0\end{array}\right)$ & 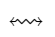 & $(0$ & -1 & 2 & 0) & $\left(\begin{array}{lllll}0 & 0 & 1 & 0 & 0\end{array}\right)$ & sur & $(0$ & -1 & 2 & -1 & $-1)$ \\
\hline$\left(\begin{array}{llll}0 & 0 & 0 & 1\end{array}\right)$ & sur & $(0$ & -1 & 0 & 2) & $\left(\begin{array}{lllll}0 & 0 & 0 & 1 & 0\end{array}\right)$ & sut & $(0$ & 0 & -1 & 2 & 0) \\
\hline$\left(\begin{array}{llll}1 & 1 & 0 & 0\end{array}\right)$ & $\leftrightarrow x$ & $(1$ & 1 & -1 & $-1)$ & $\left(\begin{array}{lllll}0 & 0 & 0 & 0 & 1\end{array}\right)$ & that & $(0$ & 0 & -1 & 0 & 2) \\
\hline$\left(\begin{array}{llll}0 & 1 & 1 & 0\end{array}\right)$ & sur & $(-1$ & 1 & 1 & $-1)$ & $\left(\begin{array}{lllll}1 & 1 & 0 & 0 & 0\end{array}\right)$ & tha & $(1$ & 1 & -1 & 0 & 0) \\
\hline$\left(\begin{array}{llll}0 & 1 & 0 & 1\end{array}\right)$ & $\leftrightarrow x$ & $(-1$ & 1 & -1 & 1) & $\left(\begin{array}{lllll}0 & 1 & 1 & 0 & 0\end{array}\right)$ & tha & $(-1$ & 1 & 1 & -1 & $-1)$ \\
\hline$\left(\begin{array}{llll}1 & 1 & 1 & 0\end{array}\right)$ & turs & $(1$ & 0 & 1 & $-1)$ & $\left(\begin{array}{lllll}0 & 0 & 1 & 1 & 0\end{array}\right)$ & tha & $(0$ & -1 & 1 & 1 & $-1)$ \\
\hline$\left(\begin{array}{llll}1 & 1 & 0 & 1\end{array}\right)$ & turs & $(1$ & 0 & -1 & 1) & $\left(\begin{array}{lllll}0 & 0 & 1 & 0 & 1\end{array}\right)$ & tha & $(0$ & -1 & 1 & -1 & 1) \\
\hline$\left(\begin{array}{llll}0 & 1 & 1 & 1\end{array}\right)$ & $\leftrightarrow x$ & $(-1$ & 0 & 1 & 1) & $\left(\begin{array}{lllll}1 & 1 & 1 & 0 & 0\end{array}\right)$ & that & $(1$ & 0 & 1 & -1 & $-1)$ \\
\hline$\left(\begin{array}{llll}1 & 1 & 1 & 1\end{array}\right)$ & $\leftrightarrow$ & $(1$ & -1 & 1 & 1) & $\left(\begin{array}{lllll}0 & 1 & 1 & 1 & 0\end{array}\right)$ & that & $(-1$ & 1 & 0 & 1 & $-1)$ \\
\hline$\left(\begin{array}{llll}1 & 2 & 1 & 1\end{array}\right)$ & $\leftrightarrow x \rightarrow$ & $(0$ & 1 & 0 & 0) & $\left(\begin{array}{lllll}0 & 0 & 1 & 1 & 1\end{array}\right)$ & thr & $(0$ & -1 & 0 & 1 & 1) \\
\hline & & & & & & $\left(\begin{array}{lllll}0 & 1 & 1 & 0 & 1\end{array}\right)$ & tha & $(-1$ & 1 & 0 & -1 & 1) \\
\hline & & & & & & $\left(\begin{array}{lllll}1 & 1 & 1 & 1 & 0\end{array}\right)$ & that & $(1$ & 0 & 0 & 1 & $-1)$ \\
\hline & & & & & & $\left(\begin{array}{lllll}0 & 1 & 1 & 1 & 1\end{array}\right)$ & that & $(-1$ & 1 & -1 & 1 & 1) \\
\hline & & & & & & $\left(\begin{array}{lllll}1 & 1 & 1 & 0 & 1\end{array}\right)$ & tha & $(1$ & 0 & 0 & -1 & 1) \\
\hline & & & & & & $\left(\begin{array}{lllll}1 & 1 & 1 & 1 & 1\end{array}\right)$ & tha & $(1$ & 0 & -1 & 1 & 1) \\
\hline & & & & & & $\left(\begin{array}{lllll}0 & 1 & 2 & 1 & 1\end{array}\right)$ & tha & $(-1$ & 0 & 1 & 0 & 0) \\
\hline & & & & & & $\left(\begin{array}{lllll}1 & 1 & 2 & 1 & 1\end{array}\right)$ & that & $(1$ & -1 & 1 & 0 & 0) \\
\hline & & & & & & $\left(\begin{array}{lllll}1 & 2 & 2 & 1 & 1\end{array}\right)$ & $\leftrightarrow$ & $(0$ & 1 & 0 & 0 & 0) \\
\hline
\end{tabular}

Let $G$ be a classical group (other than $D_{n}$ type). We prove the lemma for the higher rank groups. Similar proof holds for $D_{n}$ type also. Let $G$ be of rank $\geq 4$. Note that the weights of $\Lambda^{d-1} \mathfrak{n}^{-} \otimes k_{\lambda}$ are $\alpha-\alpha_{n-2}-\alpha_{n-2}, \alpha \in \Phi^{+}$. Let $\alpha=\beta+\gamma$, where $\beta$ involves simple roots $\alpha_{1}, \ldots, \alpha_{n-4}$ and $\gamma$ involves simple roots $\alpha_{n}, \alpha_{n-1}, \alpha_{n-2}, \alpha_{n-3}$. Let $v \in\left\{\alpha_{n-2}, \alpha_{n-1}, \alpha_{n-2}+\alpha_{n-1}\right\}$ then $\left(\beta, v^{\vee}\right)=0$. Now $\left(\alpha-\alpha_{n-2}-\alpha_{n-2}+\rho, v^{\vee}\right)=$ $\left(\beta, v^{\vee}\right)+\left(\gamma-\alpha_{n-2}-\alpha_{n-2}+\rho, v^{\vee}\right)$. We could 'think of' $\gamma$ as positive root of rank $\leq 4$ group, which proves the lemma. (In the case of type $B_{n}$, for the root $\alpha=\alpha_{1}+$ $2 \alpha_{2}+2 \alpha_{3}+\cdots+2 \alpha_{n}, \gamma$ will be $2 \alpha_{n-3}+2 \alpha_{n-2}+2 \alpha_{n-1}+2 \alpha_{n}$, which will not be a root of $B_{4}$, but we can easily see that for $v=\alpha_{n-2}+\alpha_{n-1},\left(\gamma-\alpha_{n-2}-\alpha_{n-1}+\rho, v^{\vee}\right)$ $=0$.)

Theorem 12. Let $G$ be an algebraic group with $\operatorname{rank}(G) \geq 2$. Let $\lambda$ be as given in the above lemma. Then $H^{d-1,1}(G / B, \mathscr{L}(\lambda)) \neq 0$.

Proof. Arrange the weights of $\Lambda^{d-1} \mathfrak{n}^{-} \otimes k_{\lambda}$ as $\lambda_{1}, \ldots, \lambda_{d}$ such that if $s<t$ then $\lambda_{t} \not \subset \lambda_{s}$; in other words, $\lambda_{s}$ is the lowest weight in the set of weights $\left\{\lambda_{s+1}, \lambda_{s+2}, \ldots, \lambda_{d}\right\}$ for all $s$. There are several ways to arrange the weights with the said property. Note that the weights of $\Lambda^{d-1} \mathfrak{n}^{-} \otimes k_{\lambda}$ are $\alpha-\beta, \alpha \in \Phi^{+}$where $\lambda=2 \rho-\beta$. We arrange the weight as follows: $\alpha_{1}-\beta, \alpha_{2}-\beta, \ldots, \alpha_{n}-\beta, \alpha_{1}+\alpha_{2}-\beta, \alpha_{2}+\alpha_{3}-\beta, \ldots$.

If we choose $\lambda$ as given in Lemma 11, the arrangement of weights of $\Lambda^{d-1} \mathfrak{n} \otimes k_{\lambda}$ will be of the following form: .., $\mu_{1}, \mu_{2}, \ldots, 0, \ldots$ From Lemma 11, it is clear that if there are weights in the dotted places, they all will lie in $X(T)-W \cdot X(T)^{+}$. From the Borel-Weil-Bott theorem $H^{i}\left(G / B, \mathscr{L}\left(\mu_{j}\right)\right) \neq 0$ only when $i=1$, for both $j=1,2$, and $H^{i}(G / B, \mathscr{L}(0)) \neq 0$ only when $i=0$ (note that there will be one extra dominant weight contributing to $H^{0}(G / B)$ after zero weight in case of $G_{2}$, the same technique of the proof will do even in this case). $(\star)$

Let $V_{1}=\left(\Lambda^{p} \mathfrak{n}^{-} \otimes k_{\lambda}\right) / k_{\lambda_{1}}$. Then we have short exact sequence $0 \rightarrow k_{\lambda_{1}} \rightarrow \Lambda^{p} \mathfrak{n}^{-} \otimes$ $k_{\lambda} \rightarrow V_{1} \rightarrow 0$. This induces the following long exact sequence of cohomology modules.

$$
\begin{aligned}
0 & \rightarrow H^{0}\left(G / B, \mathscr{L}\left(\lambda_{1}\right)\right) \rightarrow H^{p, 0}(G / B, \mathscr{L}(\lambda)) \rightarrow H^{0}\left(G / B, \mathscr{L}\left(V_{1}\right)\right) \\
& \rightarrow H^{1}\left(G / B, \mathscr{L}\left(\lambda_{1}\right)\right) \rightarrow H^{p, 1}(G / B, \mathscr{L}(\lambda)) \rightarrow H^{1}\left(G / B, \mathscr{L}\left(V_{1}\right)\right) \\
& \rightarrow H^{2}\left(G / B, \mathscr{L}\left(\lambda_{1}\right)\right) \rightarrow H^{p, 2}(G / B, \mathscr{L}(\lambda)) \rightarrow H^{2}\left(G / B, \mathscr{L}\left(V_{1}\right)\right) \rightarrow \cdots
\end{aligned}
$$


By our arrangement of weights $\lambda_{2}$ will be the lowest weight among all the weights of $V_{1}$. Let $V_{2}=V_{1} / k_{\lambda_{2}}$. As before we will have a short exact sequence $0 \rightarrow k_{\lambda_{1}} \rightarrow V_{1} \rightarrow V_{2} \rightarrow 0$. Which will induce the long exact sequences of cohomology modules. Proceeding like this we will have several exact sequences, but by Lemma 10, we essentially need to consider only three (in case of $G_{2}$ four) long exact sequences which involve the weights $\mu_{1}, \mu_{2}, 0$. The three exact sequence are as follows:

$$
\begin{aligned}
0 & \rightarrow H^{0}\left(G / B, \mathscr{L}\left(\mu_{1}\right)\right) \rightarrow H^{0}\left(G / B, \mathscr{L}\left(V_{s}\right)\right) \rightarrow H^{0}\left(G / B, \mathscr{L}\left(V_{s+1}\right)\right) \\
& \rightarrow H^{1}\left(G / B, \mathscr{L}\left(\mu_{1}\right)\right) \rightarrow H^{1}\left(G / B, \mathscr{L}\left(V_{s}\right)\right) \rightarrow H^{1}\left(G / B, \mathscr{L}\left(V_{s+1}\right)\right) \\
& \rightarrow H^{2}\left(G / B, \mathscr{L}\left(\mu_{1}\right)\right) \rightarrow H^{2}\left(G / B, \mathscr{L}\left(V_{s}\right)\right) \rightarrow \cdots \\
0 & \rightarrow H^{0}\left(G / B, \mathscr{L}\left(\mu_{2}\right)\right) \rightarrow H^{0}\left(G / B, \mathscr{L}\left(V_{s+1}\right)\right) \rightarrow H^{0}\left(G / B, \mathscr{L}\left(V_{s+2}\right)\right) \\
& \rightarrow H^{1}\left(G / B, \mathscr{L}\left(\mu_{2}\right)\right) \rightarrow H^{1}\left(G / B, \mathscr{L}\left(V_{s+1}\right)\right) \rightarrow H^{1}\left(G / B, \mathscr{L}\left(V_{s+2}\right)\right) \\
& \rightarrow H^{2}\left(G / B, \mathscr{L}\left(\mu_{2}\right)\right) \rightarrow \cdots \\
0 & \rightarrow H^{0}(G / B, \mathscr{L}(0)) \rightarrow H^{0}\left(G / B, \mathscr{L}\left(V_{t}\right)\right) \rightarrow H^{0}\left(G / B, \mathscr{L}\left(V_{t+1}\right)\right) \\
& \rightarrow H^{1}(G / B, \mathscr{L}(0)) \rightarrow H^{1}\left(G / B, \mathscr{L}\left(V_{t}\right)\right) \rightarrow H^{1}\left(G / B, \mathscr{L}\left(V_{t+1}\right)\right) \\
& \rightarrow H^{2}(G / B, \mathscr{L}(0)) \rightarrow H^{2}\left(G / B, \mathscr{L}\left(V_{t}\right)\right) \rightarrow \cdots
\end{aligned}
$$

If we use $(\star)$ and the Borel-Weil-Bott theorem, we could easily see that $H^{1}\left(G / B, \mathscr{L}\left(V_{s}\right)\right)$ $\neq 0$. Again by Lemma 10 and the fact that weights appeared before $\mu_{1}$ are all in $X(T)-W \cdot X(T)^{+}$, we can easily see that $H^{p, 1}(G / B, \mathscr{L}(\lambda)) \neq 0$.

The following corollary is an immediate consequence of Theorem 12.

\section{COROLLARY 13.}

Let $G$ be a simple simply connected algebraic group with $\operatorname{rank}(G) \geq 2$. Then $G / B$ does not satisfy Bott vanishing property.

Remark 14. Our motivation to prove Theorem 11 was Corollary 13. This is the reason why we wanted strict dominant weights. If we just want the result for dominant weights there are families of weights for which higher cohomology survives. For example, let $G$ be of type $A_{2}$. Let $\lambda=n \omega_{1}+m \omega_{2}$. If $n=0$ and $m \geq 2$ or $n \geq 2$ and $m=0$ then $H^{2,1}(G / B, \mathscr{L}(\lambda)) \neq 0$.

Remark 15. Let $\lambda=\sum n_{k} \omega_{k}$ and $d=\operatorname{dim}(G / B)$. If $n_{k} \geq 1, \forall k$ then $L_{\lambda}$ will be ample. By Theorem 1 , for $i \geq 1$ to get $H^{d-1, i}\left(G / B, \mathscr{L}_{\lambda}\right) \neq 0$ we cannot assume all $n_{k}$ are 'bigger'. One natural candidate with all its coefficients positive is $\rho=(1, \ldots, 1)$. But we note that if $G$ is not of type $A_{2}$ all cohomology groups vanish for $\lambda=\rho$.

Theorem 16. Let $G$ be a simple, simply connected algebraic group with $\operatorname{rank}(G) \geq 2$. Then

(i) $G / B$ is not a toric variety.

(ii) $G / B$ cannot be degenerated to a smooth toric variety in such a manner that ample cone degenerates to ample cone.

Proof.

(i) Since $G / B$ is smooth, if it is a toric variety it has to satisfy Bott vanishing property (cf. [2]), which contradicts Corollary 13. 
(ii) Suppose it degenerates to a smooth toric variety such that the ample cone degenerates to the ample cone, then by [2] and semicontinuity theorem, $G / B$ will satisfy Bott vanishing property, which contradicts Corollary 13.

\section{Remark 17.}

1. The fact that $G / B$ is not a toric variety could possibly be checked by pure topological criteria, for example by computing the integral cohomology and observing that for smooth projective toric variety the integral cohomology ring is generated by the second cohomology. Since the integral cohomology of $S L(n) / B$ is generated as a ring by degree 2 elements, this criterion will not help to conclude $S L(n) / B$ is not a toric variety.

2. One could compare the rank of the connected component of automorphism group of $G / B(=G / Z(G)$ where $Z(G)$ is the center of $G)$ with the dimension of $G / B$.

3. The above methods can be used to prove Theorem 16(i) but not Corollary 13 or Theorem 16(ii).

\section{Appendix A}

The following is the set of all positive roots of exceptional groups. We are presenting them in terms of both simple roots and fundamental weights. It will be useful to order them and to apply Borel-Weil-Bott theorem. The left-hand side is the coefficient of positive roots with respect to the simple roots, and the right-hand side is with respect to the fundamental weights.

\section{A.1 Positive roots of $G_{2}$ and $F_{4}$}

\begin{tabular}{|c|c|c|c|c|c|c|c|c|}
\hline \multicolumn{3}{|c|}{$G_{2}$} & \multicolumn{6}{|c|}{$F_{4}$} \\
\hline (1 0$)$ & ( 2 & $-3)$ & $\left(\begin{array}{llll}1 & 0 & 0 & 0\end{array}\right)$ & $m$ & ( 2 & -1 & 0 & 0) \\
\hline$\left(\begin{array}{lll}0 & 1\end{array}\right)$ & $(-1$ & 2) & $\left(\begin{array}{llll}0 & 1 & 0 & 0\end{array}\right)$ & ans & $(-1$ & 2 & -2 & $0)$ \\
\hline (1 11$)$ & ( 1 & $-1)$ & $\left(\begin{array}{llll}0 & 0 & 1 & 0\end{array}\right)$ & ans & $(0$ & -1 & 2 & $-1)$ \\
\hline (12) & $(0$ & 1) & $\left(\begin{array}{lllll}0 & 0 & 0 & 1\end{array}\right)$ & $m$ & $(0$ & 0 & -1 & 2) \\
\hline (13) & $(-1$ & 3) & 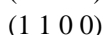 & $m$ & $(1$ & 1 & -2 & $0)$ \\
\hline (23) & ( 1 & $0)$ & $\left(\begin{array}{llll}0 & 1 & 1 & 0\end{array}\right)$ & ans & $(-1$ & 1 & 0 & $-1)$ \\
\hline & & & $\left(\begin{array}{llll}0 & 0 & 1 & 1\end{array}\right)$ & ans & ( 0 & -1 & 1 & 1) \\
\hline & & & $\left(\begin{array}{llll}1 & 1 & 1 & 0\end{array}\right)$ & $m$ & ( 1 & 0 & 0 & $-1)$ \\
\hline & & & $\left(\begin{array}{llll}0 & 1 & 1 & 1\end{array}\right)$ & ans & $(-1$ & 1 & -1 & 1) \\
\hline & & & $\left(\begin{array}{llll}1 & 1 & 1 & 1\end{array}\right)$ & $m$ & ( 1 & 0 & -1 & 1) \\
\hline & & & $\left(\begin{array}{llll}0 & 1 & 2 & 0\end{array}\right)$ & $\leftrightarrow$ & $(-1$ & 0 & 2 & $-2)$ \\
\hline & & & 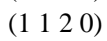 & wh & ( 1 & -1 & 2 & $-2)$ \\
\hline & & & $\left(\begin{array}{llll}0 & 1 & 2 & 1\end{array}\right)$ & ma & $(-1$ & 0 & 1 & $0)$ \\
\hline & & & 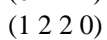 & $m$ & $(0$ & 1 & 0 & $-2)$ \\
\hline & & & $\left(\begin{array}{llll}1 & 1 & 2 & 1\end{array}\right)$ & ans & $(1$ & -1 & 1 & $0)$ \\
\hline & & & 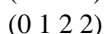 & $m$ & $(-1$ & 0 & 0 & 2) \\
\hline & & & $(1122)$ & $m$ & 1 & -1 & 0 & 2) \\
\hline & & & $\left(\begin{array}{lll}1 & 2 & 2\end{array}\right)$ & $m$ & $(0$ & 1 & -1 & $0)$ \\
\hline & & & $\left(\begin{array}{llll}1 & 2 & 2 & 2\end{array}\right)$ & ma & ( 0 & 1 & -2 & 2) \\
\hline & & & 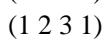 & $m$ & $(0$ & 0 & 1 & $-1)$ \\
\hline & & & 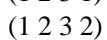 & $m$ & $(0$ & 0 & 0 & 1) \\
\hline & & & $\left(\begin{array}{lll}1 & 2 & 4\end{array}\right)$ & wh & $(0$ & -1 & 2 & 0 ) \\
\hline & & & $\left(\begin{array}{llll}1 & 3 & 4\end{array}\right)$ & $\leftrightarrow$ & $(-1$ & 1 & 0 & 0) \\
\hline & & & $(2342)$ & 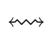 & ( 1 & 0 & 0 & 0) \\
\hline
\end{tabular}


A.2 Positive roots of $E_{6}$

\begin{tabular}{|c|c|c|c|c|c|c|c|}
\hline$\left(\begin{array}{llllll}1 & 0 & 0 & 0 & 0 & 0\end{array}\right)$ & $m$ & $(2$ & 0 & -1 & 0 & 0 & 0) \\
\hline$\left(\begin{array}{llllll}0 & 1 & 0 & 0 & 0 & 0\end{array}\right)$ & $m$ & $(0$ & 2 & 0 & -1 & 0 & $0)$ \\
\hline $\left.\begin{array}{llllllllll}0 & 0 & 0 & 0 & 0\end{array}\right)$ & $m$ & $(-1$ & 0 & 2 & -1 & 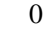 & 0) \\
\hline 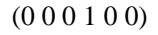 & $m$ & $(0$ & -1 & -1 & 2 & -1 & $0)$ \\
\hline $\left.\begin{array}{lllllll}0 & 0 & 0 & 1 & 0\end{array}\right)$ & $m a$ & $(0$ & 0 & 0 & -1 & 2 & $-1)$ \\
\hline$\left(\begin{array}{lllll}0 & 0 & 0 & 0 & 1\end{array}\right)$ & $m$ & $(0$ & 0 & 0 & 0 & -1 & 2) \\
\hline $\left.\begin{array}{lllll}0 & 1 & 0 & 0 & 0\end{array}\right)$ & ans & $(1$ & 0 & 1 & -1 & 0 & 0) \\
\hline 10100$)$ & $t w$ & $(0$ & 1 & -1 & 1 & -1 & $-1)$ \\
\hline $\begin{array}{llllllllll}0 & 1 & 1 & 0 & 0\end{array}$ & ans & $(-1$ & -1 & 1 & 1 & -1 & $0)$ \\
\hline$\left(\begin{array}{lllll}0 & 0 & 1 & 1 & 0\end{array}\right)$ & $m$ & $(0$ & -1 & -1 & 1 & 1 & $-1)$ \\
\hline $\left.0 \begin{array}{lllll}0 & 0 & 0 & 1 & 1\end{array}\right)$ & $m$ & $(0$ & 0 & 0 & -1 & 1 & 1) \\
\hline 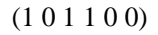 & $m$ & $(1$ & -1 & 0 & 1 & -1 & 0) \\
\hline 11100$)$ & $m$ & $(-1$ & 1 & 1 & 0 & -1 & 0) \\
\hline $\left.\begin{array}{lllll}1 & 0 & 1 & 1 & 0\end{array}\right)$ & $m$ & $(0$ & 1 & -1 & 0 & 1 & $-1)$ \\
\hline$\left(\begin{array}{lllll}0 & 1 & 1 & 1 & 0\end{array}\right)$ & ans & $(-1$ & -1 & 1 & 0 & 1 & $-1)$ \\
\hline$\left(\begin{array}{lllll}0 & 0 & 1 & 1 & 1\end{array}\right)$ & $m$ & $(0$ & -1 & -1 & 1 & 0 & 1) \\
\hline 1111100$)$ & thy & $(1$ & 1 & 0 & 0 & -1 & 0) \\
\hline$\left(\begin{array}{llllll}1 & 0 & 1 & 1 & 1 & 0\end{array}\right)$ & ans & $(1$ & -1 & 0 & 0 & 1 & $-1)$ \\
\hline$\left(\begin{array}{llllll}0 & 1 & 1 & 1 & 1 & 0\end{array}\right)$ & that & $(-1$ & 1 & 1 & -1 & 1 & $-1)$ \\
\hline$\left(\begin{array}{llllll}0 & 1 & 0 & 1 & 1 & 1\end{array}\right)$ & sus & $(0$ & 1 & -1 & 0 & 0 & 1) \\
\hline$\left(\begin{array}{lllll}0 & 1 & 1 & 1 & 1\end{array}\right)$ & $m$ & $(-1$ & -1 & 1 & 0 & 0 & 1) \\
\hline$\left(\begin{array}{llllll}1 & 1 & 1 & 1 & 1 & 0\end{array}\right)$ & $a n$ & $(1$ & 1 & 0 & -1 & 1 & $-1)$ \\
\hline 101111$)$ & $\leadsto$ & $(1$ & -1 & 0 & 0 & 0 & 1) \\
\hline 11111$)$ & $m$ & $(-1$ & 1 & 1 & -1 & 0 & 1) \\
\hline 11210$)$ & $m$ & $(-1$ & 0 & 0 & 1 & 0 & $-1)$ \\
\hline$\left(\begin{array}{llllll}1 & 1 & 1 & 1 & 1 & 1\end{array}\right)$ & tha & $(1$ & 1 & 0 & -1 & 0 & 1) \\
\hline$\left(\begin{array}{llllll}0 & 1 & 1 & 2 & 1 & 1\end{array}\right)$ & tus & $(-1$ & 0 & 0 & 1 & -1 & 1) \\
\hline$\left(\begin{array}{llllll}1 & 1 & 1 & 2 & 1 & 0\end{array}\right)$ & $m$ & $(1$ & 0 & -1 & 1 & 0 & $-1)$ \\
\hline$\left(\begin{array}{llllll}1 & 1 & 1 & 2 & 1 & 1\end{array}\right)$ & tha & $(1$ & 0 & -1 & 1 & -1 & 1) \\
\hline$\left(\begin{array}{llllll}0 & 1 & 1 & 2 & 2 & 1\end{array}\right)$ & $m$ & $(-1$ & 0 & 0 & 0 & 1 & 0) \\
\hline$\left(\begin{array}{llllll}1 & 1 & 2 & 2 & 1 & 0\end{array}\right)$ & thr & $(0$ & 0 & 1 & 0 & 0 & $-1)$ \\
\hline$\left(\begin{array}{llllll}1 & 1 & 1 & 2 & 2 & 1\end{array}\right)$ & wn & $(1$ & 0 & -1 & 0 & 1 & 0) \\
\hline$\left(\begin{array}{llllll}1 & 1 & 2 & 2 & 1 & 1\end{array}\right)$ & $m$ & $(0$ & 0 & 1 & 0 & -1 & 1) \\
\hline$\left(\begin{array}{llllll}1 & 1 & 2 & 2 & 2 & 1\end{array}\right)$ & $m a$ & $(0$ & 0 & 1 & -1 & 1 & 0) \\
\hline 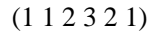 & $\leftrightarrow$ & $(0$ & -1 & 0 & 1 & 0 & 0) \\
\hline 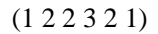 & $m$ & $(0$ & 1 & 0 & 0 & 0 & 0) \\
\hline
\end{tabular}

A.3 Positive roots of $E_{7}$

\begin{tabular}{|c|c|c|c|c|c|c|c|c|}
\hline$\left(\begin{array}{lllllll}1 & 0 & 0 & 0 & 0 & 0 & 0\end{array}\right)$ & ans & $(2$ & 0 & -1 & 0 & 0 & 0 & 0) \\
\hline 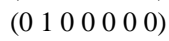 & ans & $(0$ & 2 & 0 & -1 & 0 & 0 & $0)$ \\
\hline$\left(\begin{array}{lllllll}0 & 0 & 1 & 0 & 0 & 0 & 0\end{array}\right)$ & thr & $(-1$ & 0 & 2 & -1 & 0 & 0 & ? \\
\hline$\left(\begin{array}{lllllll}0 & 0 & 0 & 1 & 0 & 0 & 0\end{array}\right)$ & tha & $(0$ & -1 & -1 & 2 & -1 & 0 & \\
\hline$\left(\begin{array}{lllllll}0 & 0 & 0 & 0 & 1 & 0 & 0\end{array}\right)$ & ans & $(0$ & 0 & 0 & -1 & 2 & -1 & \\
\hline$\left(\begin{array}{lllllll}0 & 0 & 0 & 0 & 0 & 1 & 0\end{array}\right)$ & thr & $(0$ & 0 & 0 & 0 & -1 & 2 & $-1)$ \\
\hline 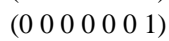 & ans & $(0$ & 0 & 0 & 0 & 0 & -1 & \\
\hline 0100000$)$ & ans & ( 1 & 0 & 1 & -1 & 0 & 0 & \\
\hline 101000$)$ & ans & $(0$ & 1 & -1 & 1 & -1 & 0 & \\
\hline 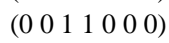 & ans & $(-1$ & -1 & 1 & 1 & -1 & 0 & \\
\hline$\left(\begin{array}{lllllll}0 & 0 & 0 & 1 & 1 & 0 & 0\end{array}\right)$ & $m a$ & $(0$ & -1 & -1 & 1 & 1 & -1 & \\
\hline 00001110$)$ & ans & $(0$ & 0 & 0 & -1 & 1 & 1 & $-1)$ \\
\hline 0000011$)$ & ans & $(0$ & 0 & 0 & 0 & -1 & 1 & \\
\hline 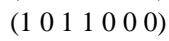 & ans & $(1$ & -1 & 0 & 1 & -1 & 0 & \\
\hline
\end{tabular}


Twisted holomorphic forms on generalized flag varieties

\begin{tabular}{|c|c|c|c|c|c|c|c|c|}
\hline$\left(\begin{array}{lllllll}0 & 1 & 1 & 1 & 0 & 0 & 0\end{array}\right)$ & $\leftrightarrow$ & $(-1$ & 1 & 1 & 0 & -1 & 0 & 0) \\
\hline$\left(\begin{array}{lllllll}0 & 1 & 0 & 1 & 1 & 0 & 0\end{array}\right)$ & $m$ & $(0$ & 1 & -1 & 0 & 1 & -1 & $0)$ \\
\hline$\left(\begin{array}{lllllll}0 & 0 & 1 & 1 & 1 & 0 & 0\end{array}\right)$ & $\leftrightarrow$ & $(-1$ & -1 & 1 & 0 & 1 & -1 & 0) \\
\hline$\left(\begin{array}{lllllll}0 & 0 & 0 & 1 & 1 & 1 & 0\end{array}\right)$ & $\leftrightarrow$ & $(0$ & -1 & -1 & 1 & 0 & 1 & $-1)$ \\
\hline$\left(\begin{array}{lllllll}0 & 0 & 0 & 0 & 1 & 1 & 1\end{array}\right)$ & ans & $(0$ & 0 & 0 & -1 & 1 & 0 & 1) \\
\hline$\left(\begin{array}{lllllll}1 & 1 & 1 & 1 & 0 & 0 & 0\end{array}\right)$ & $\leftrightarrow$ & $(1$ & 1 & 0 & 0 & -1 & 0 & $0)$ \\
\hline$\left(\begin{array}{lllllll}1 & 0 & 1 & 1 & 1 & 0 & 0\end{array}\right)$ & $\leftrightarrow$ & $(1$ & -1 & 0 & 0 & 1 & -1 & $0)$ \\
\hline$\left(\begin{array}{lllllll}0 & 1 & 1 & 1 & 1 & 0 & 0\end{array}\right)$ & ans & $(-1$ & 1 & 1 & -1 & 1 & -1 & 0) \\
\hline$\left(\begin{array}{lllllll}0 & 1 & 0 & 1 & 1 & 1 & 0\end{array}\right)$ & m & $(0$ & 1 & -1 & 0 & 0 & 1 & $-1)$ \\
\hline$\left(\begin{array}{lllllll}0 & 0 & 1 & 1 & 1 & 1 & 0\end{array}\right)$ & $\leftrightarrow$ & $(-1$ & -1 & 1 & 0 & 0 & 1 & $-1)$ \\
\hline$\left(\begin{array}{lllllll}0 & 0 & 0 & 1 & 1 & 1 & 1\end{array}\right)$ & ans & $(0$ & -1 & -1 & 1 & 0 & 0 & 1) \\
\hline$\left(\begin{array}{lllllll}1 & 1 & 1 & 1 & 1 & 0 & 0\end{array}\right)$ & $\leftrightarrow$ & $(1$ & 1 & 0 & -1 & 1 & -1 & $0)$ \\
\hline$\left(\begin{array}{lllllll}1 & 0 & 1 & 1 & 1 & 1 & 0\end{array}\right)$ & $\leftrightarrow$ & $(1$ & -1 & 0 & 0 & 0 & 1 & $-1)$ \\
\hline$\left(\begin{array}{lllllll}0 & 1 & 1 & 1 & 1 & 1 & 0\end{array}\right)$ & $m$ & $(-1$ & 1 & 1 & -1 & 0 & 1 & $-1)$ \\
\hline$\left(\begin{array}{lllllll}0 & 1 & 0 & 1 & 1 & 1 & 1\end{array}\right)$ & $\leftrightarrow$ & $(0$ & 1 & -1 & 0 & 0 & 0 & 1) \\
\hline$\left(\begin{array}{lllllll}0 & 0 & 1 & 1 & 1 & 1 & 1\end{array}\right)$ & tha & $(-1$ & -1 & 1 & 0 & 0 & 0 & 1) \\
\hline$\left(\begin{array}{lllllll}0 & 1 & 1 & 2 & 1 & 0 & 0\end{array}\right)$ & m & $(-1$ & 0 & 0 & 1 & 0 & -1 & 0) \\
\hline$\left(\begin{array}{lllllll}1 & 1 & 1 & 1 & 1 & 1 & 0\end{array}\right)$ & $\leftrightarrow$ & $(1$ & 1 & 0 & -1 & 0 & 1 & $-1)$ \\
\hline$\left(\begin{array}{lllllll}1 & 0 & 1 & 1 & 1 & 1 & 1\end{array}\right)$ & ans & $(1$ & -1 & 0 & 0 & 0 & 0 & 1) \\
\hline$\left(\begin{array}{lllllll}0 & 1 & 1 & 1 & 1 & 1 & 1\end{array}\right)$ & $m$ & $(-1$ & 1 & 1 & -1 & 0 & 0 & 1) \\
\hline$\left(\begin{array}{lllllll}0 & 1 & 1 & 2 & 1 & 1 & 0\end{array}\right)$ & $\leftrightarrow$ & $(-1$ & 0 & 0 & 1 & -1 & 1 & $-1)$ \\
\hline$\left(\begin{array}{lllllll}1 & 1 & 1 & 2 & 1 & 0 & 0\end{array}\right)$ & $\leftrightarrow$ & $(1$ & 0 & -1 & 1 & 0 & -1 & 0) \\
\hline$\left(\begin{array}{lllllll}1 & 1 & 1 & 1 & 1 & 1 & 1\end{array}\right)$ & wh & $(1$ & 1 & 0 & -1 & 0 & 0 & 1) \\
\hline$\left(\begin{array}{lllllll}1 & 1 & 1 & 2 & 1 & 1 & 0\end{array}\right)$ & ans & $(1$ & 0 & -1 & 1 & -1 & 1 & $-1)$ \\
\hline$\left(\begin{array}{lllllll}0 & 1 & 1 & 2 & 1 & 1 & 1\end{array}\right)$ & $\leftrightarrow$ & $(-1$ & 0 & 0 & 1 & -1 & 0 & 1) \\
\hline$\left(\begin{array}{lllllll}0 & 1 & 1 & 2 & 2 & 1 & 0\end{array}\right)$ & wh & $(-1$ & 0 & 0 & 0 & 1 & 0 & $-1)$ \\
\hline 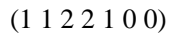 & $\leftrightarrow$ & $(0$ & 0 & 1 & 0 & 0 & -1 & 0) \\
\hline$\left(\begin{array}{lllllll}1 & 1 & 1 & 2 & 1 & 1 & 1\end{array}\right)$ & $\leftrightarrow$ & $(1$ & 0 & -1 & 1 & -1 & 0 & 1) \\
\hline$\left(\begin{array}{lllllll}1 & 1 & 1 & 2 & 2 & 1 & 0\end{array}\right)$ & $\leftrightarrow$ & $(1$ & 0 & -1 & 0 & 1 & 0 & $-1)$ \\
\hline$\left(\begin{array}{lllllll}1 & 1 & 2 & 2 & 1 & 1 & 0\end{array}\right)$ & $\leftrightarrow$ & $(0$ & 0 & 1 & 0 & -1 & 1 & $-1)$ \\
\hline$\left(\begin{array}{lllllll}0 & 1 & 1 & 2 & 2 & 1 & 1\end{array}\right)$ & $\leftrightarrow$ & $(-1$ & 0 & 0 & 0 & 1 & -1 & 1) \\
\hline$\left(\begin{array}{lllllll}1 & 1 & 2 & 2 & 1 & 1 & 1\end{array}\right)$ & $\leftrightarrow$ & $(0$ & 0 & 1 & 0 & -1 & 0 & 1) \\
\hline 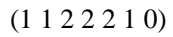 & $\leftrightarrow$ & $(0$ & 0 & 1 & -1 & 1 & 0 & $-1)$ \\
\hline$\left(\begin{array}{lllllll}1 & 1 & 1 & 2 & 2 & 1 & 1\end{array}\right)$ & ans & $(1$ & 0 & -1 & 0 & 1 & -1 & 1) \\
\hline$\left(\begin{array}{lllllll}0 & 1 & 1 & 2 & 2 & 2 & 1\end{array}\right)$ & $\leftrightarrow$ & $(-1$ & 0 & 0 & 0 & 0 & 1 & 0) \\
\hline$\left(\begin{array}{lllllll}1 & 1 & 2 & 2 & 2 & 1 & 1\end{array}\right)$ & $m$ & $(0$ & 0 & 1 & -1 & 1 & -1 & 1) \\
\hline$\left(\begin{array}{lllllll}1 & 1 & 1 & 2 & 2 & 2 & 1\end{array}\right)$ & ans & $(1$ & 0 & -1 & 0 & 0 & 1 & 0) \\
\hline 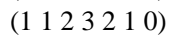 & $\leftrightarrow$ & $(0$ & -1 & 0 & 1 & 0 & 0 & $-1)$ \\
\hline$\left(\begin{array}{lllllll}1 & 1 & 2 & 2 & 2 & 2 & 1\end{array}\right)$ & $\leftrightarrow$ & $(0$ & 0 & 1 & -1 & 0 & 1 & 0) \\
\hline$\left(\begin{array}{lllllll}1 & 2 & 2 & 3 & 2 & 1 & 0\end{array}\right)$ & ans & $(0$ & 1 & 0 & 0 & 0 & 0 & $-1)$ \\
\hline$\left(\begin{array}{lllllll}1 & 1 & 2 & 3 & 2 & 1 & 1\end{array}\right)$ & uns & $(0$ & -1 & 0 & 1 & 0 & -1 & 1) \\
\hline 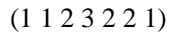 & $\leftrightarrow$ & $(0$ & -1 & 0 & 1 & -1 & 1 & 0) \\
\hline$\left(\begin{array}{lllllll}1 & 2 & 2 & 3 & 2 & 1 & 1\end{array}\right)$ & ans & $(0$ & 1 & 0 & 0 & 0 & -1 & 1) \\
\hline 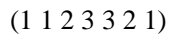 & $\leftrightarrow$ & $(0$ & -1 & 0 & 0 & 1 & 0 & 0) \\
\hline$\left(\begin{array}{llllll}1 & 2 & 2 & 32 & 2 & 1\end{array}\right)$ & ans & $(0$ & 1 & 0 & 0 & -1 & 1 & 0) \\
\hline$\left(\begin{array}{llllll}1 & 2 & 2 & 3 & 32 & 1\end{array}\right)$ & m & $(0$ & 1 & 0 & -1 & 1 & 0 & 0) \\
\hline 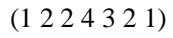 & ans & $(0$ & 0 & -1 & 1 & 0 & 0 & 0) \\
\hline 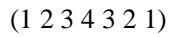 & ans & $(-1$ & 0 & 1 & 0 & 0 & 0 & 0) \\
\hline$(2234321)$ & m & $(1$ & 0 & 0 & 0 & 0 & 0 & 0) \\
\hline
\end{tabular}

\section{A.4 Positive roots of $E_{8}$}

\begin{tabular}{|c|c|c|c|c|c|c|c|c|}
\hline 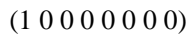 & $m$ & $(2$ & 0 & -1 & 0 & 0 & 0 & 0 \\
\hline$\left(\begin{array}{llllllll}0 & 1 & 0 & 0 & 0 & 0 & 0 & 0\end{array}\right)$ & $\leftrightarrow n$ & $(0$ & 2 & 0 & -1 & 0 & 0 & 0 \\
\hline$\left(\begin{array}{llllllll}0 & 0 & 1 & 0 & 0 & 0 & 0 & 0\end{array}\right)$ & $\leftrightarrow n$ & $(-1$ & 0 & 2 & -1 & 0 & 0 & 0 \\
\hline 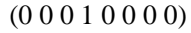 & $\leftrightarrow n$ & $(0$ & -1 & -1 & 2 & -1 & 0 & 0 \\
\hline
\end{tabular}




\begin{tabular}{|c|c|c|c|c|c|c|c|c|c|}
\hline 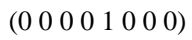 & and & $(0$ & 0 & 0 & -1 & 2 & -1 & 0 & 0) \\
\hline 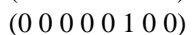 & ans & $(0$ & 0 & 0 & 0 & -1 & 2 & -1 & $0)$ \\
\hline 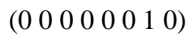 & ans & $(0$ & 0 & 0 & 0 & 0 & -1 & 2 & $-1)$ \\
\hline$\left(\begin{array}{llllllll}0 & 0 & 0 & 0 & 0 & 0 & 0 & 1\end{array}\right)$ & and & $(0$ & 0 & 0 & 0 & 0 & 0 & -1 & 2) \\
\hline$\left(\begin{array}{llllllll}1 & 0 & 1 & 0 & 0 & 0 & 0 & 0\end{array}\right)$ & ans & $(1$ & 0 & 1 & -1 & 0 & 0 & 0 & $0)$ \\
\hline$\left(\begin{array}{llllllll}0 & 1 & 0 & 1 & 0 & 0 & 0 & 0\end{array}\right)$ & ans & $(0$ & 1 & -1 & 1 & -1 & 0 & 0 & $0)$ \\
\hline$\left(\begin{array}{llllllll}0 & 0 & 1 & 1 & 0 & 0 & 0 & 0\end{array}\right)$ & ans & $(-1$ & -1 & 1 & 1 & -1 & 0 & 0 & 0) \\
\hline$\left(\begin{array}{llllllll}0 & 0 & 0 & 1 & 1 & 0 & 0 & 0\end{array}\right)$ & ans & $(0$ & -1 & -1 & 1 & 1 & -1 & 0 & 0) \\
\hline$\left(\begin{array}{llllllll}0 & 0 & 0 & 0 & 1 & 1 & 0 & 0\end{array}\right)$ & ans & $(0$ & 0 & 0 & -1 & 1 & 1 & -1 & $0)$ \\
\hline$\left(\begin{array}{llllllll}0 & 0 & 0 & 0 & 0 & 1 & 1 & 0\end{array}\right)$ & ans & $(0$ & 0 & 0 & 0 & -1 & 1 & 1 & $-1)$ \\
\hline$\left(\begin{array}{llllllll}0 & 0 & 0 & 0 & 0 & 0 & 1 & 1\end{array}\right)$ & ans & $(0$ & 0 & 0 & 0 & 0 & -1 & 1 & 1) \\
\hline 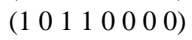 & ans & $(1$ & -1 & 0 & 1 & -1 & 0 & 0 & $0)$ \\
\hline 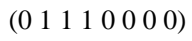 & tha & $(-1$ & 1 & 1 & 0 & -1 & 0 & 0 & 0) \\
\hline 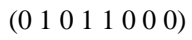 & ans & $(0$ & 1 & -1 & 0 & 1 & -1 & 0 & 0) \\
\hline$\left(\begin{array}{llllllll}0 & 0 & 1 & 1 & 1 & 0 & 0 & 0\end{array}\right)$ & ans & $(-1$ & -1 & 1 & 0 & 1 & -1 & 0 & $0)$ \\
\hline$\left(\begin{array}{llllllll}0 & 0 & 0 & 1 & 1 & 1 & 0 & 0\end{array}\right)$ & ans & $(0$ & -1 & -1 & 1 & 0 & 1 & -1 & 0) \\
\hline$\left(\begin{array}{llllllll}0 & 0 & 0 & 0 & 1 & 1 & 1 & 0\end{array}\right)$ & ans & $(0$ & 0 & 0 & -1 & 1 & 0 & 1 & $-1)$ \\
\hline$\left(\begin{array}{llllllll}0 & 0 & 0 & 0 & 0 & 1 & 1 & 1\end{array}\right)$ & ans & $(0$ & 0 & 0 & 0 & -1 & 1 & 0 & 1) \\
\hline 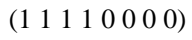 & ans & $(1$ & 1 & 0 & 0 & -1 & 0 & 0 & 0) \\
\hline 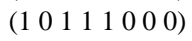 & ans & $(1$ & -1 & 0 & 0 & 1 & -1 & 0 & 0) \\
\hline 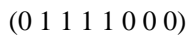 & ans & $(-1$ & 1 & 1 & -1 & 1 & -1 & 0 & $0)$ \\
\hline$\left(\begin{array}{llllllll}0 & 1 & 0 & 1 & 1 & 1 & 0 & 0\end{array}\right)$ & ans & $(0$ & 1 & -1 & 0 & 0 & 1 & -1 & $0)$ \\
\hline$\left(\begin{array}{llllllll}0 & 0 & 1 & 1 & 1 & 1 & 0 & 0\end{array}\right)$ & and & $(-1$ & -1 & 1 & 0 & 0 & 1 & -1 & $0)$ \\
\hline$\left(\begin{array}{llllllll}0 & 0 & 0 & 1 & 1 & 1 & 1 & 0\end{array}\right)$ & ans & $(0$ & -1 & -1 & 1 & 0 & 0 & 1 & $-1)$ \\
\hline 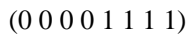 & ant & $(0$ & 0 & 0 & -1 & 1 & 0 & 0 & 1) \\
\hline 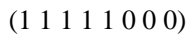 & ans & $(1$ & 1 & 0 & -1 & 1 & -1 & 0 & 0) \\
\hline$\left(\begin{array}{llllllll}1 & 0 & 1 & 1 & 1 & 1 & 0 & 0\end{array}\right)$ & ans & $(1$ & -1 & 0 & 0 & 0 & 1 & -1 & $0)$ \\
\hline$\left(\begin{array}{llllllll}0 & 1 & 1 & 1 & 1 & 1 & 0 & 0\end{array}\right)$ & turs & $(-1$ & 1 & 1 & -1 & 0 & 1 & -1 & $0)$ \\
\hline$\left(\begin{array}{lllllllll}0 & 1 & 0 & 1 & 1 & 1 & 1 & 0\end{array}\right)$ & ans & $(0$ & 1 & -1 & 0 & 0 & 0 & 1 & $-1)$ \\
\hline$\left(\begin{array}{lllllllll}0 & 0 & 1 & 1 & 1 & 1 & 1 & 0\end{array}\right)$ & ans & $(-1$ & -1 & 1 & 0 & 0 & 0 & 1 & $-1)$ \\
\hline$\left(\begin{array}{llllllll}0 & 0 & 0 & 1 & 1 & 1 & 1 & 1\end{array}\right)$ & tha & $(0$ & -1 & -1 & 1 & 0 & 0 & 0 & 1) \\
\hline 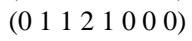 & ans & $(-1$ & 0 & 0 & 1 & 0 & -1 & 0 & 0) \\
\hline 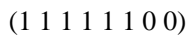 & ans & $(1$ & 1 & 0 & -1 & 0 & 1 & -1 & 0) \\
\hline$\left(\begin{array}{llllllll}1 & 0 & 1 & 1 & 1 & 1 & 1 & 0\end{array}\right)$ & ans & $(1$ & -1 & 0 & 0 & 0 & 0 & 1 & $-1)$ \\
\hline$\left(\begin{array}{lllllllll}0 & 1 & 1 & 1 & 1 & 1 & 1 & 0\end{array}\right)$ & ant & $(-1$ & 1 & 1 & -1 & 0 & 0 & 1 & $-1)$ \\
\hline$\left(\begin{array}{llllllll}0 & 1 & 0 & 1 & 1 & 1 & 1 & 1\end{array}\right)$ & ans & $(0$ & 1 & -1 & 0 & 0 & 0 & 0 & 1) \\
\hline$\left(\begin{array}{llllllll}0 & 0 & 1 & 1 & 1 & 1 & 1 & 1\end{array}\right)$ & ans & $(-1$ & -1 & 1 & 0 & 0 & 0 & 0 & 1) \\
\hline 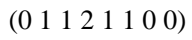 & sus & $(-1$ & 0 & 0 & 1 & -1 & 1 & -1 & 0) \\
\hline 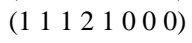 & ans & $(1$ & 0 & -1 & 1 & 0 & -1 & 0 & $0)$ \\
\hline$\left(\begin{array}{llllllll}1 & 1 & 1 & 1 & 1 & 1 & 1 & 0\end{array}\right)$ & ans & $(1$ & 1 & 0 & -1 & 0 & 0 & 1 & $-1)$ \\
\hline$\left(\begin{array}{llllllll}1 & 0 & 1 & 1 & 1 & 1 & 1 & 1\end{array}\right)$ & ans & $(1$ & -1 & 0 & 0 & 0 & 0 & 0 & 1) \\
\hline$\left(\begin{array}{lllllllll}0 & 1 & 1 & 1 & 1 & 1 & 1 & 1\end{array}\right)$ & ans & $(-1$ & 1 & 1 & -1 & 0 & 0 & 0 & 1) \\
\hline 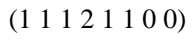 & ans & $(1$ & 0 & -1 & 1 & -1 & 1 & -1 & 0) \\
\hline$\left(\begin{array}{lllllll}0 & 1 & 1 & 2 & 1 & 1 & 1\end{array}\right)$ & ans & $(-1$ & 0 & 0 & 1 & -1 & 0 & 1 & $-1)$ \\
\hline 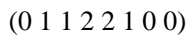 & ans & $(-1$ & 0 & 0 & 0 & 1 & 0 & -1 & $0)$ \\
\hline 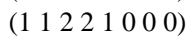 & ans & $(0$ & 0 & 1 & 0 & 0 & -1 & 0 & $0)$ \\
\hline 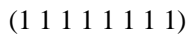 & ans & $(1$ & 1 & 0 & -1 & 0 & 0 & 0 & 1) \\
\hline$\left(\begin{array}{lllllll}1 & 1 & 1 & 2 & 1 & 1 & 1\end{array}\right)$ & ans & $(1$ & 0 & -1 & 1 & -1 & 0 & 1 & $-1)$ \\
\hline 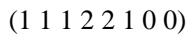 & ans & $(1$ & 0 & -1 & 0 & 1 & 0 & -1 & 0) \\
\hline 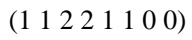 & ans & $(0$ & 0 & 1 & 0 & -1 & 1 & -1 & 0) \\
\hline$\left(\begin{array}{llllllll}0 & 1 & 1 & 2 & 1 & 1 & 1 & 1\end{array}\right)$ & ans & $(-1$ & 0 & 0 & 1 & -1 & 0 & 0 & 1) \\
\hline$\left(\begin{array}{llllllll}0 & 1 & 1 & 2 & 2 & 1 & 1 & 0\end{array}\right)$ & tuns & $(-1$ & 0 & 0 & 0 & 1 & -1 & 1 & $-1)$ \\
\hline$\left(\begin{array}{llllllll}1 & 1 & 1 & 2 & 1 & 1 & 1 & 1\end{array}\right)$ & ans & $(1$ & 0 & -1 & 1 & -1 & 0 & 0 & 1) \\
\hline$\left(\begin{array}{llllllll}0 & 1 & 1 & 2 & 2 & 1 & 1 & 1\end{array}\right)$ & ans & $(-1$ & 0 & 0 & 0 & 1 & -1 & 0 & 1) \\
\hline$\left(\begin{array}{llllllll}1 & 1 & 2 & 2 & 1 & 1 & 1 & 0\end{array}\right)$ & tuns & $(0$ & 0 & 1 & 0 & -1 & 0 & 1 & $-1)$ \\
\hline 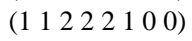 & ans & $(0$ & 0 & 1 & -1 & 1 & 0 & -1 & $0)$ \\
\hline$\left(\begin{array}{lllllll}1 & 1 & 1 & 2 & 2 & 1 & 1\end{array}\right)$ & ans & $(1$ & 0 & -1 & 0 & 1 & -1 & 1 & $-1)$ \\
\hline
\end{tabular}




\begin{tabular}{|c|c|c|c|c|c|c|c|c|c|}
\hline 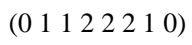 & $\leftrightarrow$ & $(-1$ & 0 & 0 & 0 & 0 & 1 & 0 & $-1)$ \\
\hline$\left(\begin{array}{llllllll}0 & 1 & 1 & 2 & 2 & 2 & 1 & 1\end{array}\right)$ & ans & $(-1$ & 0 & 0 & 0 & 0 & 1 & -1 & 1) \\
\hline$\left(\begin{array}{lllllll}1 & 1 & 22 & 1 & 1 & 1 & 1\end{array}\right)$ & ans & $(0$ & 0 & 1 & 0 & -1 & 0 & 0 & 1) \\
\hline$\left(\begin{array}{llllllll}1 & 1 & 1 & 2 & 2 & 1 & 1 & 1\end{array}\right)$ & ans & $(1$ & 0 & -1 & 0 & 1 & -1 & 0 & 1) \\
\hline$\left(\begin{array}{llllllll}1 & 1 & 2 & 2 & 2 & 1 & 1 & 0\end{array}\right)$ & ans & $(0$ & 0 & 1 & -1 & 1 & -1 & 1 & $-1)$ \\
\hline$\left(\begin{array}{llllllll}1 & 1 & 1 & 2 & 2 & 2 & 1 & 0\end{array}\right)$ & ans & $(1$ & 0 & -1 & 0 & 0 & 1 & 0 & $-1)$ \\
\hline$\left(\begin{array}{llllllll}1 & 1 & 2 & 3 & 2 & 1 & 0 & 0\end{array}\right)$ & ans & $(0$ & -1 & 0 & 1 & 0 & 0 & -1 & 0) \\
\hline$\left(\begin{array}{llllllll}1 & 1 & 2 & 2 & 2 & 1 & 1 & 1\end{array}\right)$ & ans & $(0$ & 0 & 1 & -1 & 1 & -1 & 0 & 1) \\
\hline$\left(\begin{array}{llllllll}1 & 1 & 1 & 2 & 2 & 2 & 1 & 1\end{array}\right)$ & ans & $(1$ & 0 & -1 & 0 & 0 & 1 & -1 & 1) \\
\hline$\left(\begin{array}{llllllll}0 & 1 & 1 & 2 & 2 & 2 & 2 & 1\end{array}\right)$ & $\leftrightarrow$ & $(-1$ & 0 & 0 & 0 & 0 & 0 & 1 & 0) \\
\hline 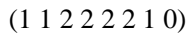 & $m$ & $(0$ & 0 & 1 & -1 & 0 & 1 & 0 & $-1)$ \\
\hline 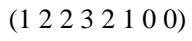 & ans & $(0$ & 1 & 0 & 0 & 0 & 0 & -1 & 0) \\
\hline$\left(\begin{array}{llllllll}1 & 1 & 2 & 3 & 2 & 1 & 1 & 0\end{array}\right)$ & ans & $(0$ & -1 & 0 & 1 & 0 & -1 & 1 & $-1)$ \\
\hline$\left(\begin{array}{lllllll}1 & 1 & 2 & 32 & 1 & 1 & 1\end{array}\right)$ & $\leftrightarrow$ & $(0$ & -1 & 0 & 1 & 0 & -1 & 0 & 1) \\
\hline$\left(\begin{array}{llllllll}1 & 1 & 2 & 2 & 2 & 2 & 1 & 1\end{array}\right)$ & ans & $(0$ & 0 & 1 & -1 & 0 & 1 & -1 & 1) \\
\hline 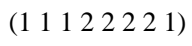 & ans & $(1$ & 0 & -1 & 0 & 0 & 0 & 1 & 0) \\
\hline$\left(\begin{array}{llllllll}1 & 1 & 2 & 3 & 2 & 2 & 1 & 0\end{array}\right)$ & ans & $(0$ & -1 & 0 & 1 & -1 & 1 & 0 & $-1)$ \\
\hline$\left(\begin{array}{llllllll}1 & 2 & 2 & 3 & 2 & 1 & 1 & 0\end{array}\right)$ & ans & $(0$ & 1 & 0 & 0 & 0 & -1 & 1 & $-1)$ \\
\hline$\left(\begin{array}{llllllll}1 & 2 & 2 & 3 & 2 & 1 & 1 & 1\end{array}\right)$ & ans & $(0$ & 1 & 0 & 0 & 0 & -1 & 0 & 1) \\
\hline 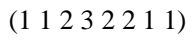 & ans & $(0$ & -1 & 0 & 1 & -1 & 1 & -1 & 1) \\
\hline 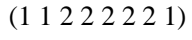 & $m$ & $(0$ & 0 & 1 & -1 & 0 & 0 & 1 & 0) \\
\hline 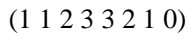 & $m$ & $(0$ & -1 & 0 & 0 & 1 & 0 & 0 & $-1)$ \\
\hline 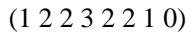 & $\leftrightarrow$ & $(0$ & 1 & 0 & 0 & -1 & 1 & 0 & $-1)$ \\
\hline 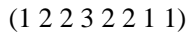 & $m$ & $(0$ & 1 & 0 & 0 & -1 & 1 & -1 & 1) \\
\hline 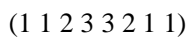 & ans & $(0$ & -1 & 0 & 0 & 1 & 0 & -1 & 1) \\
\hline 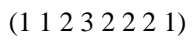 & ans & $(0$ & -1 & 0 & 1 & -1 & 0 & 1 & 0) \\
\hline 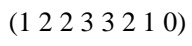 & $\leftrightarrow$ & $(0$ & 1 & 0 & -1 & 1 & 0 & 0 & $-1)$ \\
\hline 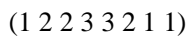 & ans & $(0$ & 1 & 0 & -1 & 1 & 0 & -1 & 1) \\
\hline 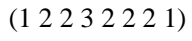 & ans & $(0$ & 1 & 0 & 0 & -1 & 0 & 1 & 0) \\
\hline 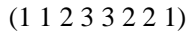 & ans & $(0$ & -1 & 0 & 0 & 1 & -1 & 1 & 0) \\
\hline 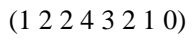 & ans & $(0$ & 0 & -1 & 1 & 0 & 0 & 0 & $-1)$ \\
\hline 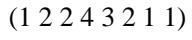 & ans & $(0$ & 0 & -1 & 1 & 0 & 0 & -1 & 1) \\
\hline 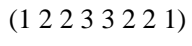 & $\leftrightarrow$ & $(0$ & 1 & 0 & -1 & 1 & -1 & 1 & 0) \\
\hline 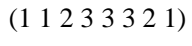 & $m$ & $(0$ & -1 & 0 & 0 & 0 & 1 & 0 & $0)$ \\
\hline$\left(\begin{array}{llll}1 & 23432 & 1 & 0\end{array}\right)$ & $m$ & $(-1$ & 0 & 1 & 0 & 0 & 0 & 0 & $-1)$ \\
\hline$(12343211)$ & $\leftrightarrow$ & $(-1$ & 0 & 1 & 0 & 0 & 0 & -1 & 1) \\
\hline$(12243221)$ & ans & $(0$ & 0 & -1 & 1 & 0 & -1 & 1 & 0) \\
\hline$\left(\begin{array}{lllllll}1 & 2 & 2 & 3 & 3 & 32 & 1\end{array}\right)$ & ans & $(0$ & 1 & 0 & -1 & 0 & 1 & 0 & 0) \\
\hline$(22343210)$ & ans & $(1$ & 0 & 0 & 0 & 0 & 0 & 0 & $-1)$ \\
\hline$(22343211)$ & $m$ & $(1$ & 0 & 0 & 0 & 0 & 0 & -1 & 1) \\
\hline$\left(\begin{array}{llllll}12 & 34322 & 1\end{array}\right)$ & ans & $(-1$ & 0 & 1 & 0 & 0 & -1 & 1 & 0) \\
\hline$\left(\begin{array}{lllll}1 & 224332 & 1\end{array}\right)$ & ans & $(0$ & 0 & -1 & 1 & -1 & 1 & 0 & 0) \\
\hline$(22343221)$ & ans & $(1$ & 0 & 0 & 0 & 0 & -1 & 1 & 0) \\
\hline$(12343321)$ & ans & $(-1$ & 0 & 1 & 0 & -1 & 1 & 0 & 0) \\
\hline$(12244321)$ & sur & $(0$ & 0 & -1 & 0 & 1 & 0 & 0 & $0)$ \\
\hline$(22343321)$ & $\leftrightarrow$ & $(1$ & 0 & 0 & 0 & -1 & 1 & 0 & $0)$ \\
\hline$(12344321)$ & $m$ & $(-1$ & 0 & 1 & -1 & 1 & 0 & 0 & 0) \\
\hline$(12354321)$ & $\leftrightarrow$ & $(-1$ & -1 & 0 & 1 & 0 & 0 & 0 & $0)$ \\
\hline$(22344321)$ & ans & $(1$ & 0 & 0 & -1 & 1 & 0 & 0 & 0) \\
\hline$(13354321)$ & ans & $(-1$ & 1 & 0 & 0 & 0 & 0 & 0 & 0) \\
\hline$(22354321)$ & ans & $(1$ & -1 & -1 & 1 & 0 & 0 & 0 & 0) \\
\hline
\end{tabular}




\begin{tabular}{|c|c|c|c|c|c|c|c|c|c|}
\hline$(23354321)$ & $m$ & $(1$ & 1 & -1 & 0 & 0 & 0 & 0 & 0) \\
\hline$(22454321)$ & $\leftrightarrow$ & $(0$ & -1 & 1 & 0 & 0 & 0 & 0 & 0) \\
\hline$(23454321)$ & $m$ & $(0$ & 1 & 1 & -1 & 0 & 0 & 0 & 0) \\
\hline$(23464321)$ & $m$ & $(0$ & 0 & 0 & 1 & -1 & 0 & 0 & 0) \\
\hline$(23465321)$ & $m$ & $(0$ & 0 & 0 & 0 & 1 & -1 & 0 & 0) \\
\hline$(23465421)$ & was & $(0$ & 0 & 0 & 0 & 0 & 1 & -1 & 0) \\
\hline$(23465431)$ & $\leadsto$ & $(0$ & 0 & 0 & 0 & 0 & 0 & 1 & $-1)$ \\
\hline$(23465432)$ & $\leftrightarrow$ & $(0$ & 0 & 0 & 0 & 0 & 0 & 0 & 1) \\
\hline
\end{tabular}

\section{Acknowledgements}

The author would like to thank Prof. C S Seshadri for his constant encouragement and V Balaji for the discussions and comments throughout the preparation of this paper. The referee's comments and suggestions (in particular the proof of Lemma 8) are gratefully acknowledged. The author also would like to thank S Senthamaraikannan and K V Subramanian for carefully reading the preliminary draft of this paper.

\section{References}

[1] Andersen H H and Jantzen J C, Cohomology of induced representations for algebraic groups, Math. Ann. 269 (1984) 487-525

[2] Anders Buch, Jesper Funch Thomsen, Niels Lauritzen and Vikram Mehta, The Frobenius morphism on a toric variety, Tohoku Math. J. (2) 49(3) (1997) 355-366

[3] Bott R, Homogeneous vector bundles, Ann. Math. 66 (1957) 203-248

[4] Jantzen J C, Representations of algebraic groups, Pure and Applied Mathematics (Florida: Academic Press) (1987) vol. 131

[5] Serre J P, Complex semisimple Lie algebras (New York: Springer-Verlag) (1987) 